\title{
Mean-Field Backward Stochastic Evolution Equations in Hilbert Spaces and Optimal Control for BSPDEs
}

\author{
Ruimin $X u^{1,2}$ and Tingting $\mathrm{Wu}^{3}$ \\ ${ }^{1}$ School of Mathematics, Shandong University, Jinan 250100, China \\ ${ }^{2}$ School of Mathematics, Shandong Polytechnic University, Jinan 250353, China \\ ${ }^{3}$ School of Mathematical Sciences, Shandong Normal University, Jinan 250014, China
}

Correspondence should be addressed to Ruimin Xu; ruiminx@126.com

Received 31 March 2014; Revised 23 May 2014; Accepted 18 June 2014; Published 13 July 2014

Academic Editor: Guangchen Wang

Copyright (C) 2014 R. Xu and T. Wu. This is an open access article distributed under the Creative Commons Attribution License, which permits unrestricted use, distribution, and reproduction in any medium, provided the original work is properly cited.

\begin{abstract}
We obtain the existence and uniqueness result of the mild solutions to mean-field backward stochastic evolution equations (BSEEs) in Hilbert spaces under a weaker condition than the Lipschitz one. As an intermediate step, the existence and uniqueness result for the mild solutions of mean-field BSEEs under Lipschitz condition is also established. And then a maximum principle for optimal control problems governed by backward stochastic partial differential equations (BSPDEs) of mean-field type is presented. In this control system, the control domain need not to be convex and the coefficients, both in the state equation and in the cost functional, depend on the law of the BSPDE as well as the state and the control. Finally, a linear-quadratic optimal control problem is given to explain our theoretical results.
\end{abstract}

\section{Introduction}

Backward stochastic evolution equations (BSEEs) in their general nonlinear form were introduced by $\mathrm{Hu}$ and Peng [1] in 1991. By the stochastic Fubini theorem and an extended martingale representation theorem, Hu and Peng [1] obtained the existence and uniqueness result of a so-called "mild solution" under Lipschitz coefficients for semilinear BSEEs. Since then, BSEEs have been studied by a lot of authors and have found various applications, namely, in the theory of infinite dimensional optimal control and the controllability for stochastic partial differential equations (see e.g., [1-4] and the papers cited therein). To relax the Lipschitz condition of the coefficients, Mahmudov and Mckibben [2] studied BSEEs under a weaker condition than the Lipschitz one in Hilbert spaces. Their approach extended the method proposed by Mao [5], in which the author investigated BSDEs under a weaker condition which contains Lipschitz condition as a special case. Our present work also investigates backward stochastic evolution equations, but with one main difference to the setting chosen by the papers mentioned above: the coefficients of the BSEEs are allowed to depend on the law of the BSEEs.

Recently, mean-field approaches, which can be used to describe particle systems at the mesoscopic level, have attracted more and more researchers' attention because of their great importance in applications. For example, meanfield approach can be used in statistical mechanics and physics, quantum mechanics and quantum chemistry, economics, finance, game theory, and optimal control theory (refer to [6-8] and the references therein). Mean-field BSDEs were deduced by Buckdahn et al. [9] when they investigated a special mean-field problem in a purely stochastic approach. Buckdahn et al. [7] studied the well posedness of mean-field BSDEs and gave a probabilistic interpretation to semilinear McKean-Vlasov partial differential equations. To give a probabilistic representation of the solutions for a class of Mckean-Vlasov stochastic partial differential equations, Xu [10] investigated the well-posedness of mean-field backward doubly stochastic differential equations with locally monotone coefficients. 
In this paper, we investigate a new type of backward stochastic evolution equations in Hilbert spaces which we call mean-field BSEEs. Mean-field implies that the coefficient of the BSEE depends on the law of the BSEE. Specifically, the BSEE we consider is defined as

$$
\begin{aligned}
d Y(s)= & -A Y(s) d s \\
- & \mathbb{E}^{\prime}\left[f\left(s, Y^{\prime}(s), Z^{\prime}(s), Y(s), Z(s)\right)\right] d s \\
+ & Z(s) d W(s), \\
& \quad Y(T)=\xi, \quad s \in[0, T]
\end{aligned}
$$

in a Hilbert space $H$, where $f$ denotes a given measurable mapping, $T$ is a fixed positive real number, $W(s)$ is a cylindrical Wiener process, and $A$ represents the generator of a strongly continuous semigroup $e^{t A}$ in $H$ with $t \geq 0$. Precise interpretation of $\mathbb{E}^{\prime}\left[f\left(s, Y^{\prime}(s), Z^{\prime}(s), Y(s), Z(s)\right)\right]$ is given in the following sections. Based on the contraction mapping, we firstly prove that (1) admits a unique mild solution if the function $f$ is Lipschitz continuous. Secondly, under non-Lipschitz assumptions, we obtain the existence and uniqueness of the mild solution for mean-field BSEE by constructing a special Cauchy sequence. The Lipschitz condition is a special case of this non-Lipschitz condition (see Mao [5]). In addition, we investigate the well-posedness of mean-field stochastic evolution equations.

We also study optimal control problems of stochastic systems governed by mean-field BSPDEs in Hilbert spaces. Our objective is to formulate a stochastic maximum principle (SMP) for the optimal control problem with an initial state constraint. There is a vast literature on the theory of SMP. Among these papers, Andersson and Djehiche [8] studied the optimal control problem for mean-field stochastic system when the control domain is convex. They obtained the maximum principle by a convex variational method. By a spike variational technique, Buckdahn et al. [11] obtained a general maximum principle for a special mean-field stochastic differential equation (SDE) where the action space is not convex. Later, Li [12] investigated the maximum principle for more general SDEs of mean-field type with a convex control domain. Wang et al. [13] were concerned with a partially observed optimal control problem of mean-field type. By using Girsanov's theorem and convex variation, they derived the corresponding maximum principle and gave an illustrative example to demonstrate the application of the obtained SMP. Hafayed studied the mean-field SMP for singular stochastic control in [14] and mean-field SMP for FBSDEs with Poisson jump processes in [15].

For the case of stochastic control systems in infinite dimensions, on the assumption that the control domain is not necessarily convex while the diffusion coefficient does not contain the control variable, $\mathrm{Hu}$ and Peng [16] used spike variation approach and Ekeland's variational principle to establish the maximum principle for semilinear stochastic evolution control systems with a final state constraint. Mahmudov and Mckibben [2] obtained an SMP for stochastic control systems governed by BSEEs in Hilbert spaces. Recently, Fuhrman et al. [17] deduced the maximum principle for optimal control of stochastic PDEs when the control domain is not necessarily convex.

We establish necessary optimality conditions for the control problem in the form of a maximum principle on the assumption that the control domain is not necessarily convex. Due to the initial state constraint, we first need to apply Ekeland's variational principle to convert the given control problem into a free initial state optimal control problem. Then spike variation approach is used to deduce the SMP in the mean-field framework. In our control system, not only the state processes which are the unique mild solution of the given BSPDE, but also the cost functional are of mean-field type. In other words, they depend on the law of the BSPDE as well as the state and the control. For this new controlled system, the adjoint equation will turn out to be a mean-field stochastic evolution equation.

The plan of this paper is organized as follows. In Section 2, we introduce some notations which are needed in what follows. In Section 3, the well-posedness of mean-field BSEE (1) is studied; we first prove the existence and uniqueness of a mild solution under the Lipschitz condition and investigate the regular dependence of the solution $(Y, Z)$ on $(\xi, f)$. And then, under the assumption that the coefficient is nonLipschitz continuous, a new result on the existence and uniqueness of the mild solution to (1) in Hilbert space is established, which generalizes the result for the Lipschitz case. Section 4 is devoted to the regularity of mean-field stochastic evolution equations. In Section 5, we derive the stochastic maximum principle for the BSPDE systems of mean-field type with an initial state constraint, and at the last part of Section 5, an LQ example is given to show the application of our maximum principle. An explicit optimal control is obtained in this example.

\section{Preliminaries}

The norm of an element $x$ in a Banach space $F$ is denoted by $|x|_{F}$ or simply $|x|$, if no confusion is possible. $\Gamma, H$, and $K$ are three real and separable Hilbert spaces. Scalar product is denoted by $\langle\cdot, \cdot\rangle$, with a subscript to specify the space, if necessary. $\mathscr{L}(\Gamma, K)$ is the space of Hilbert-Schmidt operators from $\Gamma$ to $K$, endowed with the Hilbert-Schmidt norm.

Let $(\Omega, \mathscr{F}, \mathbb{P})$ be a complete probability space. A cylindrical Wiener process $\{W(t), t \geq 0\}$ in a Hilbert space $\Gamma$ is a family of linear mappings $\Gamma \rightarrow L^{2}(\Omega, \mathscr{F}, \mathbb{P})$ such that

(i) for every $u \in \Gamma,\{W(t) u, t \geq 0\}$ is a real (continuous) Wiener process;

(ii) for every $u, v \in \Gamma$ and $t, s \geq 0, \mathbb{E}(W(t) u \cdot W(s) v)=$ $(t \wedge s)\langle u, v\rangle_{\Gamma}$.

By $\mathscr{F}_{t}, t \in[0, T]$, we denote the natural filtration of $W$, augmented with the family $\mathcal{N}$ of $\mathbb{P}$-null sets of $\mathscr{F}_{T}$ :

$$
\mathscr{F}_{t}=\sigma(W(s): s \in[0, t]) \vee \mathcal{N} .
$$

The filtration $\left(\mathscr{F}_{t}\right)_{t \geq 0}$ satisfies the usual conditions. All the concepts of measurability for stochastic processes (e.g., adapted, etc.) refer to this filtration. 
Next we define several classes of stochastic processes with values in a Hilbert space $H$.

(I) $\mathscr{H}_{\mathbb{F}}^{2}([0, T] ; H)$ denotes the set of (classes of $d \mathbb{P} \times$ $d t$ a.e. equal) measurable random processes $\left\{\psi_{t} ; t \in\right.$ $[0, T]\}$ which satisfy

(i) $\mathbb{E} \int_{0}^{T}\left|\psi_{t}\right|^{2} d t<+\infty$,

(ii) $\psi_{t}$ is $\mathscr{F}_{t}$ measurable, for a.e. $0 \leq t \leq T$.

Evidently, $\mathscr{H}_{\mathbb{F}}^{2}(0, T ; H)$ is a Banach space endowed with the canonical norm

$$
\|\psi\|=\left\{\mathbb{E} \int_{0}^{T}\left|\psi_{s}\right|^{2} d s\right\}^{1 / 2}
$$

(II) $\mathcal{S}_{\mathbb{F}}^{2}([0, T] ; H)$ denotes the set of continuous random processes $\left\{\psi_{t} ; t \in[0, T]\right\}$ which satisfy

(i) $\mathbb{E}\left(\sup _{0 \leq t \leq T}\left|\psi_{t}\right|^{2}\right)<+\infty$,

(ii) $\psi_{t}$ is $\mathscr{F}_{t}$ measurable, for a.e. $0 \leq t \leq T$.

(III) $L^{0}(\Omega, \mathscr{F}, \mathbb{P} ; H)$ denotes the space of all $H$ valued $\mathscr{F}$ measurable random variables.

(IV) For $1 \leq p<\infty, L^{p}(\Omega, \mathscr{F}, \mathbb{P} ; H)$ is the space of all $\mathscr{F}$ measurable random variables such that $\mathbb{E}\left[|\xi|^{p}\right]<\infty$.

(V) For any $\beta \in \mathbb{R}$, introduce the norm

$$
\|(y, z)\|_{\beta, t}^{2}=\mathbb{E} \int_{t}^{T} e^{2 \beta s}\left(|y(s)|^{2}+|z(s)|^{2}\right) d s
$$

on the Banach space

$$
\mathscr{K}_{\beta}[t, T]=\mathcal{S}_{\mathbb{F}}^{2}([t, T] ; H) \times \mathscr{H}_{\mathbb{F}}^{2}([t, T] ; \mathscr{L}(\Gamma, H)) .
$$

For $0<T<\infty$, all the norms $\|\cdot\|_{\beta, t}$ with different $\beta \in \mathbb{R}$ are equivalent. $\mathscr{K}[0, T]=\mathscr{K}_{0}[0, T]$ is the Banach space endowed with the norm

$$
\|(y, z)\|^{2}=\mathbb{E} \int_{0}^{T}\left(|y(s)|^{2}+|z(s)|^{2}\right) d s .
$$

The following result on BSEEs (see Lemma 2 in Mahmudov and McKibben [2]) will play a key role in proving the well-posedness of mean-field BSEEs.

Lemma 1. Let $H$ be a Hilbert space, and let $A: D(A) \subset$ $H \rightarrow H$ be a linear operator which generates a $C_{0}$-semigroup $\{S(t), 0 \leq t \leq T\}$ on $H$. For any $(\xi, f) \in L^{2}\left(\Omega, \mathscr{F}_{T}, \mathbb{P} ; H\right) \times$ $\mathscr{H}_{\mathbb{F}}^{2}([0, T], H)$ the following equation

$$
\begin{aligned}
Y(t)= & S(T-t) \xi+\int_{t}^{T} S(s-t) f(s) d s \\
& +\int_{t}^{T} S(s-t) Z(s) d W(s), \quad P \text {-a.s. }
\end{aligned}
$$

has a unique solution in $\mathscr{K}_{\beta}[0, T]$; moreover,

$$
\begin{aligned}
& \mathbb{E} \sup _{t \leq s \leq T} e^{2 \beta s}|Y(s)|^{2}+\mathbb{E} \int_{t}^{T} e^{2 \beta s}|Z(s)|^{2} d s \\
& \leq 24 M_{S}^{2}\left(e^{2 \beta T} \mathbb{E}|\xi|^{2}+\frac{1}{2 \beta} \int_{t}^{T} e^{2 \beta r} \mathbb{E}|f(r)|^{2} d r\right),
\end{aligned}
$$

where $M_{S}=\sup \left\{\|S(t)\|_{\mathfrak{B}(H)}, 0 \leq t \leq T\right\}$ and $\mathfrak{B}(H)$ is the space of bounded, linear operators on $H$.

\section{Mean-Field Backward Stochastic Evolution Equations}

In this section, we study the existence and uniqueness result of mild solutions to mean-field BSEEs in a Hilbert space $H$. To this end, we firstly recall some notations introduced by Buckdahn et al. [7].

Let $(\bar{\Omega}, \overline{\mathscr{F}}, \overline{\mathbb{P}})=(\Omega \times \Omega, \mathscr{F} \otimes \mathscr{F}, \mathbb{P} \otimes \mathbb{P})$ be the (noncompleted) product of $(\Omega, \mathscr{F}, \mathbb{P})$ with itself and we define $\overline{\mathbb{F}}=\left\{\overline{\mathscr{F}}_{t}=\mathscr{F} \otimes \mathscr{F}_{t}, 0 \leq t \leq T\right\}$ on this product space. A random variable $\xi \in L^{0}(\Omega, \mathscr{F}, \mathbb{P} ; H)$ originally defined on $\Omega$ is extended canonically to $\bar{\Omega}$ : $\xi^{\prime}\left(\omega^{\prime}, \omega\right)=$ $\xi\left(\omega^{\prime}\right),\left(\omega^{\prime}, \omega\right) \in \bar{\Omega}=\Omega \times \Omega$. For any $\eta \in L^{1}(\bar{\Omega}, \overline{\mathscr{F}}, \overline{\mathbb{P}})$, the variable $\eta(\cdot, \omega): \Omega \rightarrow K$ belongs to $L^{1}(\Omega, \mathscr{F}, \mathbb{P}), \mathbb{P}(d \omega)$ a.s., whose expectation is denoted by

$$
\mathbb{E}^{\prime}[\eta(\cdot, \omega)]=\int_{\Omega} \eta\left(\omega^{\prime}, \omega\right) \mathbb{P}\left(d \omega^{\prime}\right)
$$

Note that $\mathbb{E}^{\prime}[\eta]=\mathbb{E}^{\prime}[\eta(\cdot, \omega)] \in L^{1}(\Omega, \mathscr{F}, \mathbb{P})$ and

$$
\overline{\mathbb{E}}[\eta]\left(=\int_{\bar{\Omega}} \eta d \overline{\mathbb{P}}=\int_{\Omega} \mathbb{E}^{\prime}[\eta(\cdot, \omega)] \mathbb{P}(d \omega)\right)=\mathbb{E}\left[\mathbb{E}^{\prime}[\eta]\right]
$$

The mean-field BSEE we consider has the following form: for any given measurable mapping $f:[0, T] \times H \times \mathscr{L}(\Gamma, H) \times$ $H \times \mathscr{L}(\Gamma, H) \rightarrow H$ and $\xi \in L^{2}\left(\Omega, \mathscr{F}_{T}, \mathbb{P} ; H\right)$,

$$
\begin{aligned}
d Y(s)= & -A Y(s) d s \\
- & \mathbb{E}^{\prime}\left[f\left(s, Y^{\prime}(s), Z^{\prime}(s), Y(s), Z(s)\right)\right] d s \\
+ & Z(s) d W(s), \\
& Y(T)=\xi, \quad s \in[0, T]
\end{aligned}
$$

where $A: D(A) \subset H \rightarrow H$ is the generator of a strongly continuous semigroup $e^{t A}, t \geq 0$, in the Hilbert space $H$, with the notation $M_{A} \triangleq \sup _{t \in[0, T]}\left|e^{t A}\right|$. 
Definition 2. We say that a pair of adapted processes $(Y, Z)$ is a mild solution of mean-field $\operatorname{BSEE}$ (11) if $(Y, Z) \in$ $\mathcal{S}_{\mathbb{F}}^{2}([0, T] ; H) \times \mathscr{H}_{\mathbb{F}}^{2}([0, T] ; \mathscr{L}(\Gamma, H))$ and for all $t \in[0, T]$

$$
\begin{aligned}
Y(t)= & e^{A(T-t)} \xi \\
& +\int_{t}^{T} e^{A(s-t)} \mathbb{E}^{\prime}\left[f\left(s, Y^{\prime}(s), Z^{\prime}(s), Y(s), Z(s)\right)\right] d s \\
& -\int_{t}^{T} e^{A(s-t)} Z(s) d W(s) .
\end{aligned}
$$

Remark 3. We emphasize that the coefficient of (11) can be interpreted as

$$
\begin{aligned}
& \mathbb{E}^{\prime}[\left.f\left(s, Y^{\prime}(s), Z^{\prime}(s), Y(s), Z(s)\right)\right](\omega) \\
&= \mathbb{E}^{\prime}\left[f\left(s, Y^{\prime}(s), Z^{\prime}(s), Y(\omega, s), Z(\omega, s)\right)\right] \\
&=\int_{\Omega} f\left(\omega^{\prime}, \omega, s, Y\left(\omega^{\prime}, s\right), Z\left(\omega^{\prime}, s\right), Y(\omega, s), Z(\omega, s)\right) \\
& \quad \quad \times \mathbb{P}\left(d \omega^{\prime}\right) .
\end{aligned}
$$

3.1. Lipschitz Case. Now we study the existence and uniqueness of mild solutions to mean-field BSEE (11) under Lipschitz conditions. For $f:[0, T] \times H \times \mathscr{L}(\Gamma, H) \times H \times \mathscr{L}(\Gamma, H) \rightarrow$ $H$, assume the following.

(A1) There exists an $L>0$ such that

$$
\begin{gathered}
\left|f\left(t, y_{1}^{\prime}, z_{1}^{\prime}, y_{1}, z_{1}\right)-f\left(t, y_{2}^{\prime}, z_{2}^{\prime}, y_{2}, z_{2}\right)\right|^{2} \\
\leq L\left(\left|y_{1}^{\prime}-y_{2}^{\prime}\right|^{2}+\left|z_{1}^{\prime}-z_{2}^{\prime}\right|^{2}\right. \\
\left.\quad+\left|y_{1}-y_{2}\right|^{2}+\left|z_{1}-z_{2}\right|^{2}\right),
\end{gathered}
$$

for all $t \in[0, T], y_{i}^{\prime}, y_{i} \in H, z_{i}^{\prime}, z_{i} \in \mathscr{L}(\Gamma, H),(i=$ $1,2)$.

(A2) $f(\cdot, 0,0,0,0) \in \mathscr{H}_{\mathbb{F}}^{2}([0, T] ; H)$.

We have the following theorem.

Theorem 4. For any random variable $\xi \in L^{2}\left(\Omega, \mathscr{F}_{T}, \mathbb{P} ; H\right)$, under (A1) and (A2), mean-field BSEE (11) admits a unique mild solution $(Y, Z) \in \mathcal{S}_{\mathbb{F}}^{2}([0, T] ; H) \times \mathscr{H}_{\mathbb{F}}^{2}([0, T] ; \mathscr{L}(\Gamma, H))$.

Proof. Consider the following.

Step 1. For any $(y, z) \in \mathcal{S}_{\mathbb{F}}^{2}([0, T] ; H) \times \mathscr{H}_{\mathbb{F}}^{2}([0, T] ; \mathscr{L}(\Gamma, H))$, BSEE

$$
\begin{aligned}
Y(t)= & e^{A(T-t)} \xi \\
& +\int_{t}^{T} \mathbb{E}^{\prime}\left[e^{A(s-t)} f\left(s, y^{\prime}(s), z^{\prime}(s), Y(s), Z(s)\right)\right] d s \\
& -\int_{t}^{T} e^{A(s-t)} Z(s) d W(s), \quad 0 \leq t \leq T
\end{aligned}
$$

has a unique solution. In order to get this conclusion, we define

$$
f^{(y, z)}(s, \mu, \nu):=\mathbb{E}^{\prime}\left[f\left(s, y^{\prime}(s), z^{\prime}(s), \mu, \nu\right)\right] .
$$

Then (15) can be rewritten as

$$
\begin{aligned}
Y(t)= & e^{A(T-t)} \xi \\
& +\int_{t}^{T} e^{A(s-t)} f^{(y, z)}(Y(s), Z(s)) d s \\
& -\int_{t}^{T} e^{A(s-t)} Z(s) d W(s) .
\end{aligned}
$$

Due to (A1), for all $\left(\mu_{1}, v_{1}\right),\left(\mu_{2}, \nu_{2}\right) \in H \times \mathscr{L}(\Gamma, H), f$ satisfies

$$
\begin{aligned}
& \left|f^{(y, z)}\left(\mu_{1}, \nu_{1}\right)-f^{(y, z)}\left(\mu_{2}, \nu_{2}\right)\right|^{2} \\
& \quad \leq L\left(\left|\mu_{1}-\mu_{2}\right|^{2}+\left|\nu_{1}-v_{2}\right|^{2}\right) .
\end{aligned}
$$

According to Theorem 3.1 in [1], BSEE (15) has a unique solution.

Step 2. From Step 1, we can define a mapping $\Phi:(Y(\cdot), Z(\cdot))=$ $\Phi\left[\left(y^{\prime}(\cdot), z^{\prime}(\cdot)\right)\right]: \mathscr{K}[0, T] \rightarrow \mathscr{K}[0, T]$ through

$$
\begin{aligned}
Y(t)= & e^{A(T-t)} \xi \\
& +\int_{t}^{T} \mathbb{E}^{\prime}\left[e^{A(s-t)} f\left(s, y^{\prime}(s), z^{\prime}(s), Y(s), Z(s)\right)\right] d s \\
& -\int_{t}^{T} e^{A(s-t)} Z(s) d W(s), \quad 0 \leq t \leq T .
\end{aligned}
$$

For any $\left(y^{i}, z^{i}\right) \in \mathscr{K}[0, T]$, we set $\left(Y^{i}, Z^{i}\right)=\Phi\left[\left(y^{i}, z^{i}\right)\right], i=$ $1,2,(\bar{y}, \bar{z})=\left(y^{1}-y^{2}, z^{1}-z^{2}\right)$, and $(\bar{Y}, \bar{Z})=\left(Y^{1}-Y^{2}, Z^{1}-Z^{2}\right)$. Then, from Lemma 1, we have

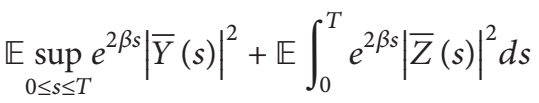

$$
\begin{aligned}
& \leq \frac{12 M_{A}^{2}}{\beta} \mathbb{E}
\end{aligned}
$$




$$
\begin{aligned}
& \times\left[\int_{0}^{T} e^{2 \beta s} \mid \mathbb{E}^{\prime}\left[f\left(s,\left(y^{1}(s)\right)^{\prime},\left(z^{1}(s)\right)^{\prime}, Y^{1}(s), Z^{1}(s)\right)\right.\right. \\
& -f\left(s,\left(y^{2}(s)\right)^{\prime},\right. \\
& \left.\left.\left.\quad\left(z^{2}(s)\right)^{\prime}, Y^{2}(s), Z^{2}(s)\right)\right]\left.\right|^{2} d s\right] \\
& \left.\left.\leq \frac{12 M_{A}^{2} L^{2}}{\beta} \mathbb{E} \quad+|\bar{Y}(s)|^{2}+|\bar{Z}(s)|^{2}\right) d s\right] \\
& \times\left[\int _ { 0 } ^ { T } e ^ { 2 \beta s } \left(\mathbb{E}\left[|\bar{y}(s)|^{2}+|\bar{z}(s)|^{2}\right]\right.\right. \\
& =\frac{12 M_{A}^{2} L^{2}}{\beta} \mathbb{E}+|\bar{z}(s)|^{2} \\
& \quad \times\left[\int_{0}^{T} e^{2 \beta s}\left(|\bar{y}(s)|^{2}+|\bar{Y}(s)|^{2}+|\bar{Z}(s)|^{2}\right) d s\right] .
\end{aligned}
$$

If we set $\beta=36 M_{A}^{2} L^{2} \max \{T, 1\}$, then

$$
\begin{aligned}
& \mathbb{E} \int_{0}^{T} e^{2 \beta s}\left(|\bar{Y}(s)|^{2}+|\bar{Z}(s)|^{2}\right) d s \\
& \leq T \cdot \sup _{0 \leq s \leq T} e^{2 \beta s}|\bar{Y}(s)|^{2}+\mathbb{E} \int_{0}^{T} e^{2 \beta s}|\bar{Z}(s)|^{2} d s \\
& \leq \frac{12 M_{A}^{2} L^{2} \max \{T, 1\}}{\beta} \mathbb{E} \\
& \times\left[\int _ { 0 } ^ { T } e ^ { 2 \beta s } \left(|\bar{y}(s)|^{2}+|\bar{z}(s)|^{2}\right.\right. \\
& \left.\left.+|\bar{Y}(s)|^{2}+|\bar{Z}(s)|^{2}\right) d s\right] \\
& =\frac{1}{3} \mathbb{E}\left[\int _ { 0 } ^ { T } e ^ { 2 \beta s } \left(|\bar{y}(s)|^{2}+|\bar{z}(s)|^{2}\right.\right. \\
& \left.\left.+|\bar{Y}(s)|^{2}+|\bar{Z}(s)|^{2}\right) d s\right] .
\end{aligned}
$$

That is,

$$
\begin{aligned}
\mathbb{E} \int_{0}^{T} e^{2 \beta s}\left(|\bar{Y}(s)|^{2}+|\bar{Z}(s)|^{2}\right) d s \\
\quad \leq \frac{1}{2} \mathbb{E} \int_{0}^{T} e^{2 \beta s}\left(|\bar{y}(s)|^{2}+|\bar{z}(s)|^{2}\right) d s .
\end{aligned}
$$

The estimate (22) shows that $\Phi$ is a contraction on the space $\mathscr{K}_{\beta}[0, T]$ with the norm

$$
\|(Y, Z)\|_{\beta}^{2}=\mathbb{E} \int_{0}^{T} e^{2 \beta s}\left(|Y(s)|^{2}+|Z(s)|^{2}\right) d s .
$$

With the contraction mapping theorem, there admits a unique fixed point $(Y, Z) \in \mathscr{K}_{\beta}[0, T]$ such that $\Phi(Y, Z)=$ $(Y, Z)$. On the other hand, from Step 1 , we know that if $\Phi(Y, Z)=(Y, Z)$, then $(Y, Z) \in \mathcal{S}_{\mathbb{F}}^{2}([0, T] ; H) \times$ $\mathscr{H}_{\mathbb{F}}^{2}([0, T] ; \mathscr{L}(\Gamma, H))$, which is the unique mild solution of (11).

Arguing as the previous proof, we arrive at the following assertion in a straightforward way.

Corollary 5. Suppose that, for all $\alpha$ in a metric space $F, f_{\alpha}$ is a given function satisfying (A1) and (A2) with $L$ independent on $\alpha$. Also suppose that

$$
\begin{aligned}
\mathbb{E}^{\prime}\left[f_{\alpha}\left(s, Y^{\prime}(s), Z^{\prime}(s), Y(s), Z(s)\right)\right] \\
\quad \longrightarrow \mathbb{E}^{\prime}\left[f_{\alpha_{0}}\left(s, Y^{\prime}(s), Z^{\prime}(s), Y(s), Z(s)\right)\right]
\end{aligned}
$$

in $L^{2}([0, T] ; H)$ as $\alpha \rightarrow \alpha_{0}$ for all $(Y, Z) \in \mathcal{S}_{\mathbb{F}}^{2}([0, T] ; H) \times$ $\mathscr{H}_{\mathbb{F}}^{2}([0, T] ; \mathscr{L}(\Gamma, H))$.

If we denote by $(Y(\xi, \alpha), Z(\xi, \alpha))$ the mild solution of (11) corresponding to the functions $f_{\alpha}$ and to the final data $\xi \in$ $L^{2}\left(\Omega, \mathscr{F}_{T}, \mathbb{P} ; H\right)$, then the map $(\alpha, \xi) \rightarrow(Y(\xi, \alpha), Z(\xi, \alpha))$ is continuous from $F \times L^{2}\left(\Omega, \mathscr{F}_{T}, \mathbb{P} ; H\right)$ to $\mathcal{S}_{\mathbb{F}}^{2}([0, T] ; H) \times$ $\mathscr{H}_{\mathbb{F}}^{2}([0, T] ; \mathscr{L}(\Gamma, H))$.

3.2. Non-Lipschitz Case. This subsection is devoted to finding some weaker conditions than the Lipschitz one under which the mean-field BSEE has a unique solution. To state our main result in this section, we suppose the following.

(A3) For all $t \in[0, T], y_{i}^{\prime}, y_{i} \in H, z_{i}^{\prime}, z_{i} \in \mathscr{L}(\Gamma, H),(i=$ $1,2)$, there exists an $l>0$, such that

$$
\begin{aligned}
&\left|f\left(t, y_{1}^{\prime}, z_{1}^{\prime}, y_{1}, z_{1}\right)-f\left(t, y_{2}^{\prime}, z_{2}^{\prime}, y_{2}, z_{2}\right)\right|^{2} \\
& \leq \theta\left(\left|y_{1}^{\prime}-y_{2}^{\prime}\right|^{2}\right)+\theta\left(\left|y_{1}-y_{2}\right|^{2}\right) \\
&+l\left(\left|z_{1}^{\prime}-z_{2}^{\prime}\right|^{2}+\left|z_{1}-z_{2}\right|^{2}\right),
\end{aligned}
$$

where $\theta: \mathbb{R}^{+} \rightarrow \mathbb{R}^{+}$is a concave increasing function such that $\theta(0)=0, \theta(u)>0$ for $u>0$ and $\int_{0^{+}}(d u / \theta(u))=\infty$.

In Mao [5], the author gave three examples of the function $\theta(\cdot)$ to show the generality of condition (A3). From these examples, we can see that Lipschitz condition (A1) is a special case of the given condition (A3).

Since $\theta$ is concave and $\theta(0)=0$, there exists a pair of positive constants $a$ and $b$ such that

$$
\theta(u) \leq a+b u
$$

for all $u \geq 0$. Therefore, under assumptions (A2) and (A3), $f\left(\cdot, y^{\prime}(\cdot), z^{\prime}(\cdot), y(\cdot), z(\cdot)\right) \in \mathscr{H}_{\mathbb{F}}^{2}([0, T] ; H)$ whenever 
$y^{\prime}(\cdot), y(\cdot) \in \mathcal{S}_{\mathbb{F}}^{2}([0, T] ; H)$ and $z^{\prime}(\cdot), z(\cdot) \in \mathscr{H}_{\mathbb{F}}^{2}([0, T] ; \mathscr{L}(\Gamma$, $H)$ ).

By Picard-type iteration, we now construct an approximate sequence, using which we obtain the desired result. Let $Y_{0}(t) \equiv 0$, and, for $n \in \mathbb{N}$, let $\left\{Y_{n}, Z_{n}\right\}$ be a sequence in $\mathcal{S}_{\mathbb{F}}^{2}([0, T] ; H) \times \mathscr{H}_{\mathbb{F}}^{2}([0, T] ; \mathscr{L}(\Gamma, H))$ defined recursively by

$$
\begin{aligned}
Y_{n}(t)= & e^{A(T-t)} \xi \\
& +\int_{t}^{T} \mathbb{E}^{\prime}\left[e^{A(s-t)}\right. \\
& \left.\quad \times f\left(s, Y_{n-1}^{\prime}(s), Z_{n}^{\prime}(s), Y_{n-1}(s), Z_{n}(s)\right)\right] d s \\
& -\int_{t}^{T} e^{A(s-t)} Z_{n}(s) d W(s),
\end{aligned}
$$

on $0 \leq t \leq T$. From Theorem 4, (27) has a unique mild solution $\left(Y_{n}(t), Z_{n}(t)\right)$.

In order to give the main result, we need to prepare the following lemmas about the properties of $\left(Y_{n}(t), Z_{n}(t)\right), t \in$ $[0, T]$.

Lemma 6. Under hypotheses (A2) and (A3), there exist positive constants $C_{1}$ and $C_{2}$ such that

$$
\begin{aligned}
& \text { (i) } \mathbb{E}\left(\sup _{t \leq s \leq T} e^{2 \beta s}\left|Y_{n}(s)\right|^{2}\right) \leq 2 C_{1} \exp (T-t), \\
& \text { (ii) } \mathbb{E} \int_{t}^{T} e^{2 \beta s}\left|Z_{n}(s)\right|^{2} d s \leq 2 C_{1} \exp (T-t), \\
& \text { (iii) } \mathbb{E}\left(\sup _{t \leq s \leq T} e^{2 \beta s}\left|Y_{n+1}(s)-Y_{n}(s)\right|^{2}\right) \\
& \quad \leq C_{2} \int_{t}^{T} \theta\left(\operatorname{Esup}_{s \leq r \leq T} e^{2 \beta r}\left|Y_{n}(r)-Y_{n-1}(r)\right|^{2}\right) d s,
\end{aligned}
$$

for all $t \in[0, T]$ and $n \geq 1$.

Proof. Using the hypotheses (A2) and (A3) with $\theta(u) \leq a+b u$ yields

$$
\begin{aligned}
\mid f(s, & \left.Y_{n-1}^{\prime}(s), Z_{n}^{\prime}(s), Y_{n-1}(s), Z_{n}(s)\right)\left.\right|^{2} \\
\leq & 2 \theta\left(\left|Y_{n-1}^{\prime}(s)\right|^{2}\right)+2 \theta\left(\left|Y_{n-1}(s)\right|^{2}\right) \\
& +2 l\left(\left|Z_{n}^{\prime}(s)\right|^{2}+\left|Z_{n}(s)\right|^{2}\right)+2|f(s, 0,0,0,0)|^{2} \\
\leq & 4 a+2 b\left|Y_{n-1}^{\prime}(s)\right|^{2}+2 b\left|Y_{n-1}(s)\right|^{2} \\
& +2 l\left(\left|Z_{n}^{\prime}(s)\right|^{2}+\left|Z_{n}(s)\right|^{2}\right)+2|f(s, 0,0,0,0)|^{2} .
\end{aligned}
$$

Then, it follows from Lemma 1 that

$$
\begin{aligned}
& \mathbb{E}\left(\sup _{t \leq s \leq T} e^{2 \beta s}\left|Y_{n}(s)\right|^{2}\right)+\mathbb{E} \int_{t}^{T} e^{2 \beta s}\left|Z_{n}(s)\right|^{2} d s \\
& \leq 24 M_{A}^{2} e^{2 \beta T} \mathbb{E}|\xi|^{2}+\frac{12 M_{A}^{2}}{\beta} \mathbb{E} \\
& \times \int_{t}^{T} e^{2 \beta s} \mid \mathbb{E}^{\prime}\left[f \left(s, Y_{n-1}^{\prime}(s),\right.\right. \\
& \left.\left.Z_{n}^{\prime}(s), Y_{n-1}(s), Z_{n}(s)\right)\right]\left.\right|^{2} d s \\
& \leq 24 M_{A}^{2} e^{2 \beta T} \mathbb{E}|\xi|^{2}+\frac{12 M_{A}^{2}}{\beta} \mathbb{E} \\
& \times \int_{t}^{T} \mathbb{E}^{\prime}\left[e^{2 \beta s} \mid f\left(s, Y_{n-1}^{\prime}(s),\right.\right. \\
& \left.\left.Z_{n}^{\prime}(s), Y_{n-1}(s), Z_{n}(s)\right)\left.\right|^{2}\right] d s \\
& \leq C_{1}+\frac{24 M_{A}^{2}}{\beta} \mathbb{E} \\
& \times \int_{t}^{T} \mathbb{E}^{\prime}\left[e ^ { 2 \beta s } \left[b\left|Y_{n-1}^{\prime}(s)\right|^{2}+b\left|Y_{n-1}(s)\right|^{2}\right.\right. \\
& \left.\left.+l\left|Z_{n}^{\prime}(s)\right|^{2}+l\left|Z_{n}(s)\right|^{2}\right]\right] d s \\
& =C_{1}+\frac{48 M_{A}^{2}}{\beta} \mathbb{E} \\
& \times \int_{t}^{T} e^{2 \beta s}\left[b\left|Y_{n-1}(s)\right|^{2}+l\left|Z_{n}(s)\right|^{2}\right] d s,
\end{aligned}
$$

where

$$
\begin{aligned}
C_{1}= & 24 M_{A}^{2} e^{2 \beta T} \mathbb{E}|\xi|^{2} \\
& +\frac{24 M_{A}^{2}}{\beta} \mathbb{E} \int_{t}^{T} e^{2 \beta s}\left[2 a+|f(s, 0,0,0,0)|^{2}\right] d s+1 .
\end{aligned}
$$

If we set $\beta=96 M_{A}^{2} \max \{b, l\}$, we can obtain

$$
\begin{aligned}
\sup _{n \in \mathbb{N}} \mathbb{E} & \left(\sup _{t \leq s \leq T} e^{2 \beta s}\left|Y_{n}(s)\right|^{2}\right)+\frac{1}{2} \int_{t}^{T} \sup _{n \in \mathbb{N}} \mathbb{E}\left[e^{2 \beta s}\left|Z_{n}(s)\right|^{2}\right] d s \\
& \leq C_{1}+\frac{1}{2} \int_{t}^{T} \sup _{n \in \mathbb{N}} \mathbb{E}\left[e^{2 \beta s}\left|Y_{n-1}(s)\right|^{2}\right] d s \\
& \leq C_{1}+\frac{1}{2} \int_{t}^{T} \sup _{n \in \mathbb{N}} \mathbb{E}\left[\sup _{s \leq r \leq T} e^{2 \beta r}\left|Y_{n-1}(r)\right|^{2}\right] d s .
\end{aligned}
$$

An application of the Gronwall inequality now implies

$$
\begin{aligned}
\sup _{n \in \mathbb{N}} \mathbb{E}\left(\sup _{t \leq s \leq T} e^{2 \beta s}\left|Y_{n}(s)\right|^{2}\right) & \leq 2 C_{1} \exp \left(\frac{T-t}{2}\right) \\
& \leq 2 C_{1} \exp (T-t) .
\end{aligned}
$$

Point (i) of Lemma 6 is now proved. 
From formula (32), we know that

$$
\begin{array}{rl}
\int_{t}^{T} \sup _{n \in \mathbb{N}} & \mathbb{E}\left[e^{2 \beta s}\left|Z_{n}(s)\right|^{2}\right] d s \\
& \leq 2 C_{1}+\int_{t}^{T} \sup _{n \in \mathbb{N}} \mathbb{E}\left[\sup _{s \leq r \leq T} e^{2 \beta r}\left|Y_{n-1}(r)\right|^{2}\right] d s \\
& \leq 2 C_{1}+2 C_{1} \int_{t}^{T} \exp (T-s) d s \\
& =2 C_{1} \exp (T-t) .
\end{array}
$$

This proves point (ii) of the Lemma.

To prove point (iii), we note that

$$
\begin{aligned}
\mathbb{E}^{\prime}\left[\mid f\left(s, Y_{n}^{\prime}(s), Z_{n+1}^{\prime}(s), Y_{n}(s), Z_{n+1}(s)\right)\right. & \left.-\left.f\left(s, Y_{n-1}^{\prime}(s), Z_{n}^{\prime}(s), Y_{n-1}(s), Z_{n}(s)\right)\right|^{2}\right] \\
\leq & \mathbb{E}^{\prime}\left[\theta\left(\left|Y_{n}^{\prime}(s)-Y_{n-1}^{\prime}(s)\right|^{2}\right)+l\left|Z_{n+1}^{\prime}(s)-Z_{n}^{\prime}(s)\right|^{2}\right] \\
& +\theta\left(\left|Y_{n}(s)-Y_{n-1}(s)\right|^{2}\right)+l\left|Z_{n+1}(s)-Z_{n}(s)\right|^{2} \\
= & \mathbb{E}\left[\theta\left(\left|Y_{n}(s)-Y_{n-1}(s)\right|^{2}\right)+l\left|Z_{n+1}(s)-Z_{n}(s)\right|^{2}\right] \\
& +\theta\left(\left|Y_{n}(s)-Y_{n-1}(s)\right|^{2}\right)+l\left|Z_{n+1}(s)-Z_{n}(s)\right|^{2} .
\end{aligned}
$$

By Lemma 1 we have

$$
\begin{gathered}
\mathbb{E}\left(\sup _{t \leq s \leq T} e^{2 \beta s}\left|Y_{n+1}(s)-Y_{n}(s)\right|^{2}\right) \\
+\mathbb{E} \int_{t}^{T} e^{2 \beta s}\left|Z_{n+1}(s)-Z_{n}(s)\right|^{2} d s \\
\leq \frac{12 M_{A}^{2}}{\beta} \mathbb{E} \\
\quad \times \int_{t}^{T} e^{2 \beta s} \mid \mathbb{E}^{\prime}\left[f\left(s, Y_{n}^{\prime}(s), Z_{n+1}^{\prime}(s), Y_{n}(s), Z_{n+1}(s)\right)\right. \\
-f\left(s, Y_{n-1}^{\prime}(s), Z_{n}^{\prime}(s),\right. \\
\left.\left.Y_{n-1}(s), Z_{n}(s)\right)\right]\left.\right|^{2} d s
\end{gathered}
$$

$$
\begin{aligned}
& \leq \frac{12 M_{A}^{2}}{\beta} \mathbb{E} \\
& \quad \times \int_{t}^{T} e^{2 \beta s} \mathbb{E}^{\prime}\left[\mid f\left(s, Y_{n}^{\prime}(s), Z_{n+1}^{\prime}(s), Y_{n}(s), Z_{n+1}(s)\right)\right. \\
& \quad-f\left(s, Y_{n-1}^{\prime}(s), Z_{n}^{\prime}(s),\right. \\
& \leq \frac{24 M_{A}^{2}}{\beta} \mathbb{E} \int_{t}^{T} e^{2 \beta s}\left[\theta\left(\left|Y_{n}(s)-Y_{n-1}(s)\right|^{2}\right)\right. \\
& \left.\quad+l\left|Z_{n+1}(s)-Z_{n}(s)\right|^{2}\right] d s .
\end{aligned}
$$

We can choose $\beta>0$ sufficiently large such that

$$
\left(1-\frac{24 M_{A}^{2} l}{\beta}\right) \mathbb{E} \int_{t}^{T} e^{2 \beta s}\left\|Z_{n+1}(s)-Z_{n}(s)\right\|^{2} d s \geq 0 .
$$

Then

$$
\begin{gathered}
\mathbb{E}\left(\sup _{t \leq s \leq T} e^{2 \beta s}\left|Y_{n+1}(s)-Y_{n}(s)\right|^{2}\right) \\
\leq \frac{24 M_{A}^{2}}{\beta} \mathbb{E} \int_{t}^{T} e^{2 \beta s} \theta\left(\left|Y_{n}(s)-Y_{n-1}(s)\right|^{2}\right) d s \\
\leq C_{2} \mathbb{E} \int_{t}^{T} \theta\left(\mathbb{E} \sup _{s \leq r \leq T} e^{2 \beta r}\left|Y_{n}(r)-Y_{n-1}(r)\right|^{2}\right) d s,
\end{gathered}
$$

where we set $C_{2}=\left(24 M_{A}^{2} / \beta\right) e^{2 \beta T}$.

We divide the interval $[0, T]$ into subintervals $0=\tau_{0}<$ $\tau_{1}<\cdots<\tau_{m}=T$ by setting $\tau_{k}=k \delta, k=1,2,3, \ldots, m$ with $\delta=T / m$.

Lemma 7. For all $t \in\left[\tau_{k-1}, \tau_{k}\right]$, define

$$
C_{3}=C_{2} \theta\left(2 C_{1} \exp (T)\right),
$$

$$
\begin{aligned}
& \varphi_{k, 1}(t)=C_{3}\left(\tau_{k}-t\right), \\
& \varphi_{k, n+1}(t)=C_{2} \int_{t}^{\tau_{k}} \theta\left(\varphi_{k, n}(s)\right) d s, \quad n \geq 1 .
\end{aligned}
$$

Then, for all $n \geq 1$, the following inequality holds for a suitable $\delta>0$ :

$$
0 \leq \varphi_{k, n}(t) \leq \varphi_{k, n-1}(t) \leq \cdots \leq \varphi_{k, 1}(t) .
$$

Proof. Firstly, it needs to be verified that for all $t \in\left[\tau_{k-1}, \tau_{k}\right]$ the following inequality

$$
\begin{aligned}
\varphi_{k, 2}(t) & =C_{2} \int_{t}^{\tau_{k}} \theta\left(\varphi_{k, 1}(s)\right) d s=C_{2} \int_{t}^{\tau_{k}} \theta\left(C_{3}\left(\tau_{k}-s\right)\right) d s \\
& \leq C_{3}\left(\tau_{k}-t\right)=\varphi_{k, 1}(t)
\end{aligned}
$$

holds provided $\delta>0$ is chosen sufficiently small.

Actually, this inequality holds provided that

$$
C_{2} \theta\left(C_{3}\left(\tau_{k}-t\right)\right) \leq C_{3}=C_{2} \theta\left(2 C_{1} \exp (T)\right)
$$


or

$$
C_{3}\left(\tau_{k}-t\right)=C_{2} \theta\left(2 C_{1} \exp (T)\right)\left(\tau_{k}-t\right) \leq 2 C_{1} \exp (T) .
$$

Since $C_{1}>1$, from $\theta(u) \leq a+b u$, the above inequality holds if

$$
C_{2}(a+b)\left(\tau_{k}-t\right) \leq 1
$$

Thus, (41) holds for any $t \in\left[\tau_{k-1}, \tau_{k}\right], k=1,2, \ldots, m$ if $\tau_{k}-$ $\tau_{k-1} \leq 1 / C_{2}(a+b)$. Therefore, we can choose a sufficiently large $m \in \mathbb{N}$ such that $\delta=T / m \leq 1 / C_{2}(a+b)$. Clearly, such a $\delta$ only depends on $a, b, l, T$, and $M_{A}$.

Now, assume that (40) holds for some $n \geq 2$. Then, we have

$$
\begin{aligned}
\varphi_{k, n+1}(t) & =C_{2} \int_{t}^{\tau_{k}} \theta\left(\varphi_{k, n}(s)\right) d s \leq C_{2} \int_{t}^{\tau_{k}} \theta\left(\varphi_{k, n-1}(s)\right) d s \\
& =\varphi_{k, n}(t), \quad \forall t \in\left[\tau_{k-1}, \tau_{k}\right] .
\end{aligned}
$$

This completes the proof.

Now, we can give the main result of this section.

Theorem 8. Assume that (A2) and (A3) hold. Then, there exists a unique mild solution $(Y, Z)$ to (11).

Proof. Consider the following.

Uniqueness. To show the uniqueness, let both $(Y, Z)$ and $(\tilde{Y}, \widetilde{Z})$ be solutions of $(11)$. For any $\beta>0$, similar to the proof of (36), one can obtain

$$
\begin{aligned}
\mathbb{E}\left(\sup _{t \leq s \leq T} e^{2 \beta s}|Y(s)-\widetilde{Y}(s)|^{2}\right)+\mathbb{E} \int_{t}^{T} e^{2 \beta s}|Z(s)-\widetilde{Z}(s)|^{2} d s \\
\leq \frac{24 M_{A}^{2}}{\beta} \mathbb{E} \\
\quad \times \int_{t}^{T} e^{2 \beta s}\left[\theta\left(|Y(s)-\tilde{Y}(s)|^{2}\right)+l|Z(s)-\widetilde{Z}(s)|^{2}\right] d s .
\end{aligned}
$$

That is, if $\beta$ is sufficiently large,

$$
\begin{aligned}
& \mathbb{E}\left(\sup _{t \leq s \leq T} e^{2 \beta s}|Y(s)-\tilde{Y}(s)|^{2}\right) \\
& \leq \frac{24 M_{A}^{2}}{\beta} \mathbb{E} \int_{t}^{T} e^{2 \beta s}\left[\theta\left(|Y(s)-\tilde{Y}(s)|^{2}\right)\right] d s \\
& \leq C_{2} \mathbb{E} \int_{t}^{T} \theta\left(\mathbb{E} \sup _{s \leq r \leq T} e^{2 \beta r}|Y(s)-\tilde{Y}(s)|^{2}\right) d s .
\end{aligned}
$$

An application of Bihari inequality yields

$$
\mathbb{E}\left(\sup _{t \leq s \leq T} e^{2 \beta s}|Y(s)-\tilde{Y}(s)|^{2}\right)=0 .
$$

So $Y(t)=\tilde{Y}(t)$ for all $t \in[0, T]$ a.s. It then follows from (46) that $Z(t)=\widetilde{Z}(t)$ for all $t \in[0, T]$ a.s. as well. This establishes the uniqueness.

Existence. We claim that the sequence $\left(Y_{n}, Z_{n}\right)$ defined by (27) satisfies

$$
\mathbb{E} \sup _{t \leq s \leq T} e^{2 \beta s}\left|Y_{n+1}(s)-Y_{n}(s)\right|^{2} \longrightarrow 0, \quad \forall 0 \leq t \leq T,
$$

as $n \rightarrow \infty$.

Indeed, for all $t \in\left[\tau_{k-1}, \tau_{k}\right]$, we set $\tilde{\varphi}_{k, n}(t)=$ $\mathbb{E} \sup _{s \in\left[t, \tau_{k}\right]} e^{2 \beta s}\left|Y_{n+1}(s)-Y_{n}(s)\right|^{2}$. By Lemmas 6 and 7,

$$
\begin{aligned}
\widetilde{\varphi}_{k, 1}(t) & =\mathbb{E} \sup _{s \in\left[t, \tau_{k}\right]} e^{2 \beta s}\left|Y_{2}(s)-Y_{1}(s)\right|^{2} \\
& \leq C_{2} \int_{t}^{\tau_{k}} \theta\left(\mathbb{E} \sup _{s \leq r \leq \tau_{k}} e^{2 \beta r}\left|Y_{1}(r)-Y_{0}(r)\right|^{2}\right) d s \\
& \leq C_{2} \int_{t}^{\tau_{k}} \theta\left(2 C_{1} \exp \left(\tau_{k}-t\right)\right) d s \\
& \leq C_{2} \theta\left(2 C_{1} \exp (T)\right)\left(\tau_{k}-t\right)=C_{3}\left(\tau_{k}-t\right)=\varphi_{k, 1}(t) .
\end{aligned}
$$

Suppose that $\widetilde{\varphi}_{k, n}(t) \leq \varphi_{k, n}(t)$ holds for some $n \geq 1$. According to Lemma 6(iii) and Lemma 7, for all $t \in\left[\tau_{k-1}, \tau_{k}\right]$, we obtain

$$
\begin{aligned}
\tilde{\varphi}_{k, n+1}(t) & =\mathbb{E} \sup _{s \in\left[t, \tau_{k}\right]} e^{2 \beta s}\left|Y_{n+2}(s)-Y_{n+1}(s)\right|^{2} \\
& \leq C_{2} \int_{t}^{\tau_{k}} \theta\left(\mathbb{E} \sup _{r \in\left[s, \tau_{k}\right]} e^{2 \beta r}\left|Y_{n+1}(r)-Y_{n}(r)\right|^{2}\right) d s \\
& =C_{2} \int_{t}^{\tau_{k}} \theta\left(\tilde{\varphi}_{k, n}(s)\right) d s \\
& \leq C_{2} \int_{t}^{\tau_{k}} \theta\left(\varphi_{k, n}(s)\right) d s=\varphi_{k, n+1}(t)
\end{aligned}
$$

This implies that, for all $n \in \mathbb{N}$,

$$
\widetilde{\varphi}_{k, n}(t) \leq \varphi_{k, n}(t) .
$$

By definition, $\varphi_{k, n}(\cdot)$ is continuous on $\left[\tau_{k-1}, \tau_{k}\right]$. Note that for each $n \geq 1, \varphi_{k, n}(\cdot)$ is decreasing on $\left[\tau_{k-1}, \tau_{k}\right]$, and for each $t, k, \varphi_{k, n}(t)$ is a nonincreasing sequence. Therefore, we define the function $\varphi_{k}(t)$ by $\varphi_{k, n}(t) \downarrow \varphi_{k}(t)$. It is easy to verify that $\varphi_{k}(t)$ is continuous and nonincreasing on $\left[\tau_{k-1}, \tau_{k}\right]$. By the definitions of $\varphi_{k, n}(t)$ and $\varphi_{k}(t)$ we get

$$
\varphi_{k}(t)=\lim _{n \rightarrow \infty} C_{2} \int_{t}^{\tau_{k}} \theta\left(\varphi_{k, n}(s)\right) d s=C_{2} \int_{t}^{\tau_{k}} \theta\left(\varphi_{k}(s)\right) d s
$$

for all $\tau_{k-1} \leq t \leq \tau_{k}$. Since $\int_{0^{+}}(d u / \theta(u))=\infty$, the Bihari inequality implies

$$
\varphi_{k}(t)=0, \quad \text { for each } t \in\left[\tau_{k-1}, \tau_{k}\right] .
$$

For each $k \in\{1,2, \ldots, m\},(52)$ and (54) yield

$$
\lim _{n \rightarrow \infty} \widetilde{\varphi}_{k, n}(t)=0 \text {. }
$$


Then,

$$
\begin{aligned}
& \mathbb{E} \sup _{t \leq s \leq T} e^{2 \beta s}\left|Y_{n+1}(s)-Y_{n}(s)\right|^{2} \\
& \leq \max _{1 \leq k \leq m} \mathbb{E} \sup _{s \in\left[\tau_{k-1}, \tau_{k}\right]} e^{2 \beta s}\left|Y_{n+1}(s)-Y_{n}(s)\right|^{2} \\
& =\max _{1 \leq k \leq m} \widetilde{\varphi}_{k, n}(t) \longrightarrow 0,
\end{aligned}
$$

as $n \rightarrow \infty$, and this proves the assertion (49).

By (36), we obtain

$$
\begin{aligned}
\mathbb{E}\left(\sup _{t \leq s \leq T} e^{2 \beta s}\left|Y_{n+1}(s)-Y_{n}(s)\right|^{2}\right)+\left(1-\frac{24 M_{A}^{2} l}{\beta}\right) \mathbb{E} \\
\quad \times \int_{t}^{T} e^{2 \beta s}\left|Z_{n+1}(s)-Z_{n}(s)\right|^{2} d s \\
\leq \frac{24 M_{A}^{2}}{\beta} \mathbb{E} \int_{t}^{T} e^{2 \beta s}\left[\theta\left(\left|Y_{n}(s)-Y_{n-1}(s)\right|^{2}\right)\right] d s .
\end{aligned}
$$

Applying (49) to the above formula, we see that $\left(Y_{n}, Z_{n}\right)$ is a Cauchy (hence convergent) sequence in $\delta_{\mathbb{F}}^{2}([0, T] ; H) \times$ $\mathscr{H}_{\mathbb{F}}^{2}([0, T] ; \mathscr{L}(\Gamma, H))$; denote the limit by $(Y, Z)$. Now letting $n \rightarrow \infty$ in (27), we obtain that

$$
\begin{aligned}
Y(t)= & e^{A(T-t)} \xi \\
& +\int_{t}^{T} \mathbb{E}^{\prime}\left[e^{A(s-t)} f\left(s, Y^{\prime}(s), Z^{\prime}(s), Y(s), Z(s)\right)\right] d s \\
& -\int_{t}^{T} e^{A(s-t)} Z(s) d W(s)
\end{aligned}
$$

holds on the entire interval $[0, T]$. The theorem is now proved.

To illustrate the application of the obtained existence and uniqueness result, we consider the example of backward stochastic partial differential equations (BSPDEs) of meanfield type.

Example 9. Let $\mathcal{O}$ be an open bounded domain in $\mathbb{R}^{n}$ with uniformly $C^{2}$ boundary $\partial \mathcal{O}$, let $B(t)$ be a standard $n$ dimensional Brownian motion (equipped with the normal filtration), and let $\xi: \mathcal{O} \rightarrow \mathbb{R}$ be an $\mathscr{F}_{T}$-measurable random variable. We also let $L$ denote the semielliptic partial differential operator on $C^{2}(\mathbb{R})$ of the form

$$
L=\sum_{i, j=1}^{n} a_{i j}(x) \frac{\partial^{2}}{\partial x_{i} \partial x_{j}}+\sum_{i=1}^{n} b_{i}(x) \frac{\partial}{\partial x_{i}}
$$

The aim is to study the solvability of the following initial boundary value problem:

$$
\begin{aligned}
& d Y(t, x) \\
& =\left(L Y(t, x)+\mathbb{E}^{\prime}\right. \\
& \left.\times\left[g\left(t, x, Y^{\prime}(t, x), Z^{\prime}(t, x), Y(t, x), Z(t, x)\right)\right]\right) d t \\
& +Z(t, x) d B(t), \quad \text { a.e. on }(0, T) \times \mathcal{O} \\
& Y(t, x)=0, \quad \text { a.e. on }(0, T) \times \partial \mathcal{O}, \\
& Y(T, x)=\xi(T, x), \quad \text { a.e. on } \mathcal{O},
\end{aligned}
$$

where

$$
\begin{aligned}
& Y:[0, T] \times \mathcal{O} \longrightarrow \mathbb{R}, \\
& Z:[0, T] \times \mathcal{O} \longrightarrow \mathscr{L}\left(\mathbb{R}^{n} ; L^{2}(\mathcal{O})\right), \\
& g:[0, T] \times \mathcal{O} \times \mathbb{R} \times \mathscr{L}\left(\mathbb{R}^{n} ; L^{2}(\mathcal{O})\right) \\
& \quad \times \mathbb{R} \times \mathscr{L}\left(\mathbb{R}^{n} ; L^{2}(\mathcal{O})\right) \longrightarrow \mathbb{R} .
\end{aligned}
$$

The following assumptions will have to be in force.

(H1) $a_{i j}, b_{i}: \mathbb{R}^{n} \rightarrow \mathbb{R}$ are uniformly continuous and bounded and satisfy the usual uniform ellipticity condition: $\sum_{i, j=1}^{n} a_{i j}(x) w_{i} w_{j} \geq \lambda|w|^{2}$, for some $\lambda>0$ and all $x \in \overline{\mathcal{O}}, w \in \mathbb{R}^{n}$.

(H2) $g$ is measurable in $(t, x, \tilde{y}, \tilde{z}, y, z)$ and continuous in $(\widetilde{z}, z)$, and there exists $C>0$ such that

$$
\begin{aligned}
& \left|g\left(t, x, \tilde{y}_{1}, \widetilde{z}_{1}, y_{1}, z_{1}\right)-g\left(t, x, \tilde{y}_{2}, \widetilde{z}_{2}, y_{2}, z_{2}\right)\right| \\
& \quad \leq C\left[\left|\tilde{y}_{1}-\tilde{y}_{2}\right|+\left|\widetilde{z}_{1}-\widetilde{z}_{2}\right|+\left|y_{1}-y_{2}\right|+\left|z_{1}-z_{2}\right|\right],
\end{aligned}
$$

for all $0 \leq t \leq T, x \in \mathcal{O}, \tilde{y}_{1}, \tilde{y}_{2}, y_{1}, y_{2} \in \mathbb{R}, \widetilde{z}_{1}, \widetilde{z}_{2}, z_{1}, z_{2} \in$ $\mathscr{L}\left(\mathbb{R}^{n} ; L^{2}(\mathcal{O})\right)$.

Then, we are now in a position of showing existence and uniqueness of the solution of BSPDEs (60).

Theorem 10. If (H1) and (H2) are satisfied, then the meanfield BSPDE (60) has a unique mild solution $(Y, Z) \in$ $L^{2}\left(0, T ; L^{2}\left(\Omega, L^{2}(\mathcal{O})\right)\right) \times L_{\mathbb{F}}^{2}\left(0, T ; L^{2}\left(\mathbb{R}^{n}, L^{2}\left(\Omega, L^{2}(\mathcal{O})\right)\right)\right)$.

Proof. Let $H=L^{2}(\mathcal{O})$ and $K=\mathbb{R}^{n}$. Define the operator $A$ by

$$
A Y(t, \cdot)=L Y(t, \cdot) .
$$

It is shown in [17] (see Example 2.1 in [17]) that $A$ generates a strongly continuous semigroup on $H$. Define the maps $f$ : $[0, T] \times H \times \mathscr{L}(K, H) \times H \times \mathscr{L}(K, H) \rightarrow H$ by

$$
\begin{aligned}
& f\left(t, Y^{\prime}(t), Z^{\prime}(t), Y(t), Z(t)\right)(x) \\
& \quad=g\left(t, x, Y^{\prime}(t, x), Z^{\prime}(t, x), Y(t, x), Z(t, x)\right)
\end{aligned}
$$


for all $0 \leq t \leq T, x \in \mathcal{O}$. With these identifications, (60) can be written in the form of (11). By (H2), we know $f$ satisfy condition (A1). Hence, an application of Theorem 4 concludes that $(60)$ has a unique mild solution $(Y, Z) \in$ $L^{2}\left(0, T ; L^{2}\left(\Omega, L^{2}(\mathcal{O})\right)\right) \times L_{\mathbb{F}}^{2}\left(0, T ; L^{2}\left(\mathbb{R}^{n}, L^{2}\left(\Omega, L^{2}(\mathcal{O})\right)\right)\right)$.

\section{Mean-Field Stochastic Evolution Equations}

Let $W(t), t \in[0, T]$, be a cylindrical Wiener process with values in a Hilbert space $\Gamma$, defined on a probability space $(\Omega, \mathscr{F}, \mathbb{P})$. We fix an interval $[t, T] \subset[0, T]$ and consider the stochastic evolution equations of mean-field type for an unknown process $X(s), s \in[t, T]$ with values in a Hilbert space $K$ :

$$
\begin{gathered}
d X(s)=B X(s) d s+\mathbb{E}^{\prime}\left[b\left(s, X^{\prime}(s), X(s)\right)\right] d s \\
+\mathbb{E}^{\prime}\left[\sigma\left(s, X^{\prime}(s), X(s)\right)\right] d W(s), \\
X(t)=x \in K,
\end{gathered}
$$

where operator $B$ is the generator of a strongly continuous semigroup $e^{t B}, t \geq 0$, in the Hilbert space $K$, with $M_{B} \triangleq$ $\sup _{t \in[0, T]}\left|e^{t B}\right|$.

By a mild solution of (65) we mean an $\mathscr{F}_{s}$-measurable process $X(s), s \in[t, T]$, with continuous paths in $K$, such that, $\mathbb{P}$-a.s.,

$$
\begin{aligned}
& X(s)= e^{B(s-t)} x \\
&+\int_{t}^{s} e^{B(s-\tau)} \mathbb{E}^{\prime}\left[b\left(\tau, X^{\prime}(\tau), X(\tau)\right)\right] d \tau \\
&+\int_{t}^{s} e^{B(s-\tau)} \mathbb{E}^{\prime}\left[\sigma\left(\tau, X^{\prime}(\tau), X(\tau)\right)\right] d W(\tau), \\
& s \in[t, T] .
\end{aligned}
$$

We suppose the following.

(A4) $b:[0, T] \times K \times K \rightarrow K$ is a measurable mapping which satisfies

$$
\begin{aligned}
& \left|b\left(t, x^{\prime}, x\right)-b\left(t, y^{\prime}, y\right)\right|^{2} \\
& \quad \leq L_{1}\left(\left|x^{\prime}-y^{\prime}\right|^{2}+|x-y|^{2}\right), \\
& \quad t \in[0, T], x^{\prime}, x, y^{\prime}, y \in K,
\end{aligned}
$$

for some constant $L_{1}>0$.

(A5) The mapping $\sigma:[0, T] \times K \times K \rightarrow \mathscr{L}(\Gamma, K)$ fulfills that for every $v \in \Gamma$ the map $\sigma v:[0, T] \times K \times K \rightarrow K$ is measurable, for every $s>0, t \in[0, T], x^{\prime}, y^{\prime}, x, y \in K$, $e^{s B} \sigma\left(t, x^{\prime}, x\right) \in \mathscr{L}(\Gamma, K)$, and

$$
\begin{gathered}
\left|e^{s B} \sigma\left(t, x^{\prime}, x\right)\right|_{\mathscr{L}(\Gamma, K)} \leq L_{2} s^{-\gamma}\left(1+\left|x^{\prime}\right|+|x|\right), \\
\left|e^{s B} \sigma\left(t, x^{\prime}, x\right)-e^{s B} \sigma\left(t, y^{\prime}, y\right)\right|_{\mathscr{L}(\Gamma, K)} \\
\leq L_{2} s^{-\gamma}\left(\left|x^{\prime}-y^{\prime}\right|+|x-y|\right) \\
\left|\sigma\left(t, x^{\prime}, x\right)\right|_{\mathscr{L}(\Gamma, K)} \leq L_{2}\left(1+\left|x^{\prime}\right|+|x|\right),
\end{gathered}
$$

for some constants $L_{2}>0$ and $\gamma \in[0,1 / 2)$.
Theorem 11. Under assumptions (A3) and (A4), (65) has a unique mild solution $X(\cdot) \in \mathcal{S}_{\mathbb{F}}^{2}([t, T] ; K)$.

The proof is constructed in two steps like that of Theorem 4 and it uses standard arguments for stochastic evolution equations introduced in the proof of Proposition 3.2 in [3]. Since the proof is straightforward, we prefer to omit it.

Remark 12. In our paper, Lipchitz condtion (A4) is given to get the well-posedness of mean-field stochastic evolution equations. In fact, (A4) can be replaced by a weaker condition such as (A3). We just give the condition (A4) for simplicity.

From standard arguments, we can also get the following continuous dependence theorem.

Corollary 13. Assume that for all $\alpha$ in a metric space $F$, $\left(b_{\alpha}, \sigma_{\alpha}\right)$ satisfy $(A 4)$ and (A5) with $L_{1}$ and $L_{2}$ independent of $\alpha$. Also assume that

$$
\begin{aligned}
& \mathbb{E} \int_{0}^{T} \mid \mathbb{E}^{\prime}\left[b_{\alpha}\left(s, X^{\prime}(s), X(s)\right)\right]-\left.\mathbb{E}^{\prime}\left[b_{\alpha_{0}}\left(s, X^{\prime}(s), X(s)\right)\right]\right|^{2} d s \longrightarrow 0, \\
& \mathbb{E} \int_{0}^{T} \mid \mathbb{E}^{\prime}\left[\sigma_{\alpha}\left(s, X^{\prime}(s), X(s)\right)\right] \\
& \quad-\left.\mathbb{E}^{\prime}\left[\sigma_{\alpha_{0}}\left(s, X^{\prime}(s), X(s)\right)\right]\right|^{2} d s \longrightarrow 0,
\end{aligned}
$$

as $\alpha \rightarrow \alpha_{0}$ for all $X \in \mathcal{S}_{\mathbb{F}}^{2}([0, T] ; K)$.

If we denote by $X^{\alpha}(\cdot)$ the mild solution of mean-field SEE (65) corresponding to the functions $\left(b_{\alpha}, \sigma_{\alpha}\right)$ and to the initial data $x$, then we have

$$
\sup _{s \in[0, T]} \mathbb{E}\left|X^{\alpha}(s)-X^{\alpha_{0}}(s)\right|^{2} \longrightarrow 0, \quad \text { as } \alpha \longrightarrow \alpha_{0} .
$$

\section{Maximum Principle for BSPDEs of Mean-Field Type}

5.1. Formulation of the Problem. Let $\mathcal{O} \in \mathbb{R}^{n}$ be a bounded open set with smooth boundary $\partial \mathcal{O}$ and let $U$, the space of controls, be a separable real Hilbert space. We denote

$$
\begin{aligned}
\mathscr{U}=\left\{v(\cdot) \in L_{\mathscr{F}}^{2}\right. & (0, T ; U) \\
& \mid v_{t}\left(\omega^{\prime}, \omega\right):[0, T] \times \Omega \times \Omega
\end{aligned}
$$

$\longrightarrow U$ is $\mathscr{F} \otimes \mathscr{F}_{t}$-progressively measurable $\}$. 
An element of $\mathcal{U}$ is called an admissible control.

For any $v \in \mathcal{U}$, we consider the following controlled BSPDE system in the state space $H=L^{2}(\mathcal{O})$ (norm $|\cdot|$, scalar product $\langle\cdot, \cdot\rangle)$ :

$$
\begin{aligned}
& d Y_{t}(x)=-A Y_{t}(x) d t \\
& -\mathbb{E}^{\prime}\left[f \left(t, x,\left(Y_{t}(x)\right)^{\prime},\left(Z_{t}(x)\right)^{\prime},\right.\right. \\
& \left.\left.\quad Y_{t}(x), Z_{t}(x), v_{t}\right)\right] d t \\
& +Z_{t}(x) d W(t), \quad t \in[0, T], \\
& Y_{T}(x)=\xi(x), \quad x \in \mathcal{O},
\end{aligned}
$$

where $A$ is a partial differential operator, $f:[0, T] \times \mathcal{O} \times H \times$ $\mathscr{L}(\Gamma, H) \times H \times \mathscr{L}(\Gamma, H) \times U \rightarrow H$, and $\xi \in L^{2}\left(\Omega, \mathscr{F}_{T}, \mathbb{P} ; H\right)$.

The cost functional is given by

$$
\begin{gathered}
J(v)=\mathbb{E}\left\{\int _ { 0 } ^ { T } \int _ { \mathcal { O } } \mathbb { E } ^ { \prime } \left[h \left(s, x,\left(Y_{s}(x)\right)^{\prime},\left(Z_{s}(x)\right)^{\prime},\right.\right.\right. \\
\left.\left.Y_{s}(x), Z_{s}(x), v_{s}\right)\right] d x d s \\
\left.+\mathbb{E}^{\prime} \int_{\mathcal{O}} g\left(x,\left(Y_{0}(x)\right)^{\prime}, Y_{0}(x)\right) d x\right\},
\end{gathered}
$$

where

$$
\begin{gathered}
h:[0, T] \times \mathcal{O} \times H \times \mathscr{L}(\Gamma, H) \times H \times \mathscr{L}(\Gamma, H) \times U \longrightarrow \mathbb{R}, \\
g: \mathcal{O} \times H \times H \longrightarrow \mathbb{R} .
\end{gathered}
$$

Our purpose is to minimize the functional $J(\cdot)$ over $\mathscr{U}_{\mathrm{ad}}$, subject to the following state constraint:

$$
\overline{\mathbb{E}} \int_{\mathscr{O}} \Phi\left(x,\left(Y_{0}(x)\right)^{\prime}, Y_{0}(x)\right) d x=0,
$$

where

$$
\Phi: \mathcal{O} \times H \times H \longrightarrow \mathbb{R}
$$

An admissible control $\bar{u} \in \mathscr{U}_{\text {ad }}$ that satisfies

$$
J(\bar{u})=\min _{v \in \mathscr{U}_{\mathrm{ad}}} J(v)
$$

is called optimal.

Through what follows, the following assumptions will be in force.

(L1) $A$ is a partial differential operator with appropriate boundary conditions. We assume that $A$ is the infinitesimal generator of a strongly continuous semigroup $e^{t A}, t \geq 0$ in $H$. Moreover, for every $t \in[0, T]$, $\left\|e^{t A} f\right\|_{L^{2}(\mathcal{O})} \leq M_{A}\|f\|_{L^{2}(\mathcal{O})}$ for some constant $M_{A}$ independent of $t$ and $f$.

(L2) $f, h, g$, and $\Phi$ are continuously Gâteaux differentiable with respect to $\left(y^{\prime}, z^{\prime}, y, z\right) . f$ is continuously Gâteaux differentiable with respect to $v$ and $h$ is continuous with respect to $v$.
(L3) The derivatives of $f, h, g$, and $\Phi$ are Lipschitz continuous and bounded by

$$
\begin{gathered}
\left|f_{y^{\prime}}\right|+\left|f_{z^{\prime}}\right|+\left|f_{y}\right|+\left|f_{z}\right|+\left|f_{v}\right|+\left|\Phi_{y^{\prime}}\right|+\left|\Phi_{y}\right| \leq C, \\
\left|h_{y^{\prime}}\right|+\left|h_{z^{\prime}}\right|+\left|h_{y}\right|+\left|h_{z}\right|+\left|g_{y^{\prime}}\right|+\left|g_{y}\right| \\
\leq C\left(1+\left|y^{\prime}\right|+\left|z^{\prime}\right|+|y|+|z|+|v|\right),
\end{gathered}
$$

where $C$ is a positive constant.

Obviously, according to Theorem 4, state equation (72) has a unique mild solution under the above assumptions.

Remark 14. We can define the second order differential operator:

$$
(A f)(x)=\sum_{i, j=1}^{n} a_{i j}(x) \frac{\partial^{2} f}{\partial x_{i} \partial x_{j}}(x)+\sum_{i=1}^{n} b_{i}(x) \frac{\partial f}{\partial x_{i}}(x) .
$$

By Example 9, $A$ fulfills assumption (L1) if $a_{i j}, b_{i}$ satisfy condition (H1).

5.2. Variation of the Trajectory. Let $\bar{u}$ be an optimal control with $(\bar{Y}(\cdot), \bar{Z}(\cdot))$ being the corresponding optimal state. Let $\varepsilon>0$ and $[r, r+\varepsilon] \subseteq[0, T]$. For any given $v \in \mathcal{U}_{\mathrm{ad}}$, we introduce the spike variation of the control $\bar{u}(\cdot)$ :

$$
u_{t}^{\varepsilon}= \begin{cases}v_{t}, & t \in[r, r+\varepsilon], \\ \bar{u}_{t}, & t \in[0, T] \backslash[s, s+\varepsilon] .\end{cases}
$$

It is clear that $u^{\varepsilon}(\cdot) \in \mathcal{U}_{\mathrm{ad}}$.

Let $\left(Y^{\varepsilon}(\cdot), Z^{\varepsilon}(\cdot)\right)$ be the trajectory corresponding to $u^{\varepsilon}(\cdot)$. We use the following short notation for brevity:

$$
\begin{aligned}
& f(t)=f\left(t, x,\left(\bar{Y}_{t}(x)\right)^{\prime},\left(\bar{Z}_{t}(x)\right)^{\prime}, \bar{Y}_{t}(x), \bar{Z}_{t}(x), \bar{u}_{t}\right), \\
& f\left(u_{t}^{\varepsilon}\right)=f\left(t, x,\left(\bar{Y}_{t}(x)\right)^{\prime},\left(\bar{Z}_{t}(x)\right)^{\prime}, \bar{Y}_{t}(x), \bar{Z}_{t}(x), u_{t}^{\varepsilon}\right) .
\end{aligned}
$$

Consider the following equation:

$$
\begin{gathered}
d K_{t}^{\varepsilon}(x)=-A K_{t}^{\varepsilon}(x) d t \\
-\mathbb{E}^{\prime}\left[f_{y^{\prime}}(t)\left(K_{t}^{\varepsilon}(x)\right)^{\prime}+f_{y}(t) K_{t}^{\varepsilon}(x)\right. \\
+f_{z^{\prime}}(t)\left(Q_{t}^{\varepsilon}(x)\right)^{\prime}+f_{z}(t) Q_{t}^{\varepsilon}(x) \\
\left.+\frac{1}{\varepsilon}\left(f\left(u_{t}^{\varepsilon}\right)-f(t)\right)\right] d t+Q_{t}^{\varepsilon}(x) d W(t), \\
K_{T}^{\varepsilon}(x)=0 .
\end{gathered}
$$

Since the coefficients in (82) are bounded, it is easy to check that there exists a unique mild solution such that

$$
\mathbb{E}\left[\sup _{t \in[0, T]}\left|K_{t}^{\varepsilon}\right|^{2}+\int_{0}^{T}\left|Q_{t}^{\varepsilon}\right|^{2} d t\right]<\infty
$$

We have the following estimate. 
Theorem 15. There holds

$$
\begin{gathered}
\lim _{\varepsilon \rightarrow 0} \mathbb{E}\left[\sup _{s \in[t, T]}\left|\frac{Y_{s}^{\varepsilon}-\bar{Y}_{s}}{\varepsilon}-K_{s}^{\varepsilon}\right|^{2}\right]=0, \quad \forall t \in[0, T], \\
\lim _{\varepsilon \rightarrow 0} \mathbb{E} \int_{0}^{T}\left|\frac{Z_{s}^{\varepsilon}-\bar{Z}_{s}}{\varepsilon}-Q_{s}^{\varepsilon}\right|^{2} d s=0 .
\end{gathered}
$$

Proof. We define

$$
\eta_{s}^{\varepsilon}=\frac{Y_{s}^{\varepsilon}-\bar{Y}_{s}}{\varepsilon}-K_{s}^{\varepsilon}, \quad \zeta_{s}^{\varepsilon}=\frac{Z_{s}^{\varepsilon}-\bar{Z}_{s}}{\varepsilon}-Q_{s}^{\varepsilon}, \quad s \in[0, T] .
$$

For simplicity, let us define

$$
\begin{gathered}
\Lambda_{s}^{\varepsilon}=\left(\left(Y_{s}^{\varepsilon}\right)^{\prime},\left(Z_{s}^{\varepsilon}\right)^{\prime}, Y_{s}^{\varepsilon}, Z_{s}^{\varepsilon}\right), \\
f(s, \lambda)=f\left(s, \bar{Y}_{s}^{\prime}+\lambda\left(Y_{s}^{\varepsilon}-\bar{Y}_{s}\right)^{\prime},\right. \\
\bar{Z}_{s}^{\prime}+\lambda\left(Z_{s}^{\varepsilon}-\bar{Z}_{s}\right)^{\prime}, \bar{Y}_{s}+\lambda\left(Y_{s}^{\varepsilon}-\bar{Y}_{s}\right), \\
\left.\bar{Z}_{s}+\lambda\left(Z_{s}^{\varepsilon}-\bar{Z}_{s}\right), u_{s}^{\varepsilon}\right) .
\end{gathered}
$$

By the definition of $\left(Y_{s}^{\varepsilon}, Z_{s}^{\varepsilon}\right),\left(\bar{Y}_{s}, \bar{Z}_{s}\right)$, and $\left(K_{s}^{\varepsilon}, Q_{s}^{\varepsilon}\right),\left(\eta_{s}^{\varepsilon}, \zeta_{s}^{\varepsilon}\right)$ is the mild solution of

$$
\begin{gathered}
d \eta_{s}^{\varepsilon}=-A \eta_{s}^{\varepsilon} d s-\mathbb{E}^{\prime}[L(s, \varepsilon)] d s+\zeta_{s}^{\varepsilon} d W(s), \\
\eta_{T}^{\varepsilon}=0
\end{gathered}
$$

with

$$
\begin{aligned}
L(s, \varepsilon)= & \frac{1}{\varepsilon}\left(f\left(s, \Lambda_{s}^{\varepsilon}, u_{s}^{\varepsilon}\right)-f\left(u_{s}^{\varepsilon}\right)\right) \\
& -f_{y^{\prime}}(s)\left(K_{s}^{\varepsilon}\right)^{\prime}-f_{y}(s) K_{s}^{\varepsilon}-f_{z^{\prime}}(s)\left(Q_{s}^{\varepsilon}\right)^{\prime}-f_{z}(s) Q_{s}^{\varepsilon} \\
= & \left(\left(\eta_{s}^{\varepsilon}\right)^{\prime}+\left(K_{s}^{\varepsilon}\right)^{\prime}\right) \int_{0}^{1} f_{y^{\prime}}(s, \lambda) d \lambda+\left(\eta_{s}^{\varepsilon}+K_{s}^{\varepsilon}\right) \\
& \times \int_{0}^{1} f_{y}(s, \lambda) d \lambda-f_{y^{\prime}}(s)\left(K_{s}^{\varepsilon}\right)^{\prime}-f_{y}(s) K_{s}^{\varepsilon} \\
& +\left(\left(\zeta_{s}^{\varepsilon}\right)^{\prime}+\left(Q_{s}^{\varepsilon}\right)^{\prime}\right) \int_{0}^{1} f_{z^{\prime}}(s, \lambda) d \lambda+\left(\zeta_{s}^{\varepsilon}+Q_{s}^{\varepsilon}\right) \\
& \times \int_{0}^{1} f_{z}(s, \lambda) d \lambda-f_{z^{\prime}}(s)\left(Q_{s}^{\varepsilon}\right)^{\prime}-f_{z}(s) Q_{s}^{\varepsilon} \\
= & \left(\eta_{s}^{\varepsilon}\right)^{\prime} \int_{0}^{1} f_{y^{\prime}}(s, \lambda) d \lambda+\eta_{s}^{\varepsilon} \int_{0}^{1} f_{y}(s, \lambda) d \lambda \\
& +\left(\zeta_{s}^{\varepsilon}\right)^{\prime} \int_{0}^{1} f_{z^{\prime}}(s, \lambda) d \lambda+\zeta_{s}^{\varepsilon} \int_{0}^{1} f_{z}(s, \lambda) d \lambda+\gamma_{s}^{\varepsilon}
\end{aligned}
$$

where we denote

$$
\begin{aligned}
\gamma_{s}^{\varepsilon}= & \left(K_{s}^{\varepsilon}\right)^{\prime} \int_{0}^{1}\left(f_{y^{\prime}}(s, \lambda)-f_{y^{\prime}}(s)\right) d \lambda \\
& +K_{s}^{\varepsilon} \int_{0}^{1}\left(f_{y}(s, \lambda)-f_{y}(s)\right) d \lambda \\
& +\left(Q_{s}^{\varepsilon}\right)^{\prime} \int_{0}^{1}\left(f_{z^{\prime}}(s, \lambda)-f_{z^{\prime}}(s)\right) d \lambda \\
& +Q_{s}^{\varepsilon} \int_{0}^{1}\left(f_{z}(s, \lambda)-f_{z}(s)\right) d \lambda .
\end{aligned}
$$

For any $\beta>0$, according to Lemma 1 , we obtain

$$
\begin{aligned}
\underset{t \leq s \leq T}{\mathbb{E} \sup _{t} e^{2 \beta s}\left|\eta_{s}^{\varepsilon}\right|^{2}+\mathbb{E} \int_{t}^{T} e^{2 \beta s}\left|\zeta_{s}^{\varepsilon}\right|^{2} d s} \\
\leq \frac{12 M_{A}^{2}}{\beta} \int_{t}^{T} e^{2 \beta s} \mathbb{E}\left[\left|\mathbb{E}^{\prime}[L(s, \varepsilon)]\right|^{2}\right] d s \\
\leq \frac{12 M_{A}^{2}}{\beta} \mathbb{E} \int_{t}^{T} e^{2 \beta s} \mathbb{E}^{\prime}\left[|L(s, \varepsilon)|^{2}\right] d s .
\end{aligned}
$$

By condition (L3), we have

$$
\begin{aligned}
\mathbb{E}[ & \left.\mathbb{E}^{\prime}\left[|L(s, \varepsilon)|^{2}\right]\right] \\
= & \mathbb{E}\left[\mathbb{E}^{\prime}\left[\left|L(s, \varepsilon)-\gamma_{s}^{\varepsilon}+\gamma_{s}^{\varepsilon}\right|^{2}\right]\right] \\
\leq & 8 C^{2} \mathbb{E}\left[\mathbb{E}^{\prime}\left[\left|\left(\eta_{s}^{\varepsilon}\right)^{\prime}\right|^{2}+\left|\eta_{s}^{\varepsilon}\right|^{2}+\left|\left(\zeta_{s}^{\varepsilon}\right)^{\prime}\right|^{2}+\left|\zeta_{s}^{\varepsilon}\right|^{2}\right]\right] \\
& +2 \mathbb{E}\left[\mathbb{E}^{\prime}\left[\left|\gamma_{s}^{\varepsilon}\right|^{2}\right]\right] \\
\leq & 16 C^{2} \mathbb{E}\left[\left|\eta_{s}^{\varepsilon}\right|^{2}+\left|\zeta_{s}^{\varepsilon}\right|^{2}\right]+2 \mathbb{E}\left[\mathbb{E}^{\prime}\left[\left|\gamma_{s}^{\varepsilon}\right|^{2}\right]\right] .
\end{aligned}
$$

Combined with (91), (90) yields

$$
\begin{aligned}
& \mathbb{E} \sup _{t \leq s \leq T} e^{2 \beta s}\left|\eta_{s}^{\varepsilon}\right|^{2}+\left(1-\frac{192 M_{A}^{2} C^{2}}{\beta}\right) \mathbb{E} \int_{t}^{T} e^{2 \beta s}\left|\zeta_{s}^{\varepsilon}\right|^{2} d s \\
& \leq \frac{192 M_{A}^{2} C^{2}}{\beta} \mathbb{E} \int_{t}^{T} e^{2 \beta s}\left|\eta_{s}^{\varepsilon}\right|^{2} d s \\
& +\frac{24 M_{A}^{2}}{\beta} \mathbb{E} \int_{t}^{T} e^{2 \beta s}\left[\mathbb{E}^{\prime}\left[\left|\gamma_{s}^{\varepsilon}\right|^{2}\right]\right] d s
\end{aligned}
$$

We claim that

$$
\mathbb{E} \int_{t}^{T} \mathbb{E}^{\prime}\left[\left|\gamma_{s}^{\varepsilon}\right|^{2}\right] d s \longrightarrow 0, \quad \text { as } \varepsilon \longrightarrow 0
$$

From (89)

$$
\gamma_{s}^{\varepsilon}=I_{s}^{1}+I_{s}^{2}+I_{s}^{3}+I_{s}^{4},
$$


where

$$
\begin{aligned}
& I_{s}^{1}=\left(K_{s}^{\varepsilon}\right)^{\prime} \int_{0}^{1}\left(f_{y^{\prime}}(s, \lambda)-f_{y^{\prime}}(s)\right) d \lambda, \\
& I_{s}^{2}=K_{s}^{\varepsilon} \int_{0}^{1}\left(f_{y}(s, \lambda)-f_{y}(s)\right) d \lambda, \\
& I_{s}^{3}=\left(Q_{s}^{\varepsilon}\right)^{\prime} \int_{0}^{1}\left(f_{z^{\prime}}(s, \lambda)-f_{z^{\prime}}(s)\right) d \lambda, \\
& I_{s}^{4}=Q_{s}^{\varepsilon} \int_{0}^{1}\left(f_{z}(s, \lambda)-f_{z}(s)\right) d \lambda .
\end{aligned}
$$

Then,

$$
\begin{aligned}
\mathbb{E} \int_{t}^{T} & \mathbb{E}^{\prime}\left[\left|\gamma_{s}^{\varepsilon}\right|^{2}\right] d s \\
& \leq 4 \mathbb{E} \int_{t}^{T} \mathbb{E}^{\prime}\left[\left|I_{s}^{1}\right|^{2}+\left|I_{s}^{2}\right|^{2}+\left|I_{s}^{3}\right|^{2}+\left|I_{s}^{4}\right|^{2}\right] d s .
\end{aligned}
$$

Take $\mathbb{E} \int_{t}^{T} \mathbb{E}^{\prime}\left[\left|I_{s}^{2}\right|^{2}\right] d s$; for example,

$$
\begin{aligned}
\mathbb{E} \int_{t}^{T} \mathbb{E}^{\prime}\left[\left|I_{s}^{2}\right|^{2}\right] d s \\
=\mathbb{E} \int_{t}^{T} \mathbb{E}^{\prime}\left[\left(K_{s}^{\varepsilon} \int_{0}^{1}\left(f_{y}(s, \lambda)-f_{y}(s)\right) d \lambda\right)^{2}\right] d s \\
\leq \mathbb{E} \int_{t}^{T} \mathbb{E}^{\prime}\left[\left|K_{s}^{\varepsilon}\right|^{2} \int_{0}^{1}\left|f_{y}(s, \lambda)-f_{y}(s)\right|^{2} d \lambda\right] d s \\
\leq 2 \mathbb{E} \int_{t}^{T} \mathbb{E}^{\prime}\left[\left|K_{s}^{\varepsilon}\right|^{2} \int_{0}^{1}\left|f_{y}(s, \lambda)-f_{y}\left(u_{t}^{\varepsilon}\right)\right|^{2} d \lambda\right] d s \\
\quad+2 \mathbb{E} \int_{t}^{T} \mathbb{E}^{\prime}\left[\left|K_{s}^{\varepsilon}\right|^{2} \int_{0}^{1}\left|f_{y}\left(u_{t}^{\varepsilon}\right)-f_{y}(s)\right|^{2} d \lambda\right] d s .
\end{aligned}
$$

Note that

$$
\begin{aligned}
& \mathbb{E} \int_{t}^{T} \mathbb{E}^{\prime}\left[\left|K_{s}^{\varepsilon}\right|^{2} \int_{0}^{1}\left|f_{y}\left(u_{t}^{\varepsilon}\right)-f_{y}(s)\right|^{2} d \lambda\right] d s \\
& \quad=\mathbb{E} \int_{r}^{r+\varepsilon} \mathbb{E}^{\prime}\left[\left|K_{s}^{\varepsilon}\right|^{2} \int_{0}^{1}\left|f_{y}\left(u_{t}^{\varepsilon}\right)-f_{y}(s)\right|^{2} d \lambda\right] d s \\
& \quad \leq \sup _{s \in[t, T]} \mathbb{E}\left[\mathbb{E}^{\prime}\left[\left|K_{s}^{\varepsilon}\right|^{2} \int_{0}^{1}\left|f_{y}\left(u_{t}^{\varepsilon}\right)-f_{y}(s)\right|^{2} d \lambda\right]\right] \varepsilon \\
& \quad \longrightarrow 0, \quad \text { as } \varepsilon \longrightarrow 0 .
\end{aligned}
$$

The inequality above holds due to the boundedness of $\left|K_{s}^{\varepsilon}\right|^{2} \int_{0}^{1}\left|f_{y}\left(u_{t}^{\varepsilon}\right)-f_{y}(s)\right|^{2} d \lambda$. Indeed, Assumption (L3) implies the boundedness of $f_{y}\left(u_{t}^{\varepsilon}\right)-f_{y}(s)$. Meanwhile $K_{s}^{\varepsilon}$ is the solution of mean-field BSEE (82). It can be easy to check $K_{s}^{\varepsilon}$ is bounded since the coefficients in (82) are bounded.

On the other hand,

$$
\begin{aligned}
\mathbb{E} \int_{t}^{T} \mathbb{E}^{\prime}\left[\left|K_{s}^{\varepsilon}\right|^{2} \int_{0}^{1}\left|f_{y}(s, \lambda)-f_{y}\left(u_{t}^{\varepsilon}\right)\right|^{2} d \lambda\right] d s \\
\leq C^{2} \lambda^{2} \mathbb{E} \int_{t}^{T}\left|K_{s}^{\varepsilon}\right|^{2} \\
\times \int_{0}^{1} E^{\prime}\left[\left|\left(Y_{s}^{\varepsilon}-\bar{Y}_{s}\right)^{\prime}\right|^{2}+\left|\left(Z_{s}^{\varepsilon}-\bar{Z}_{s}\right)^{\prime}\right|^{2}\right. \\
\left.\times\left|Y_{s}^{\varepsilon}-\bar{Y}_{s}\right|^{2}+\left|Z_{s}^{\varepsilon}-\bar{Z}_{s}\right|^{2}\right] d \lambda d s,
\end{aligned}
$$

where $\left(Y_{s}^{\varepsilon}, Z_{s}^{\varepsilon}\right)$ is the mild solution of the following equation:

$$
\begin{aligned}
d Y_{t}^{\varepsilon}= & -A Y_{t}^{\varepsilon} d t \\
& -\mathbb{E}^{\prime}\left[f\left(t,\left(Y_{t}^{\varepsilon}\right)^{\prime},\left(Z_{t}^{\varepsilon}\right)^{\prime}, Y_{t}^{\varepsilon}, Z_{t}^{\varepsilon}, u_{t}^{\varepsilon}\right)\right] d t \\
& +Z_{t}^{\varepsilon} d W(t), \quad t \in[0, T],
\end{aligned}
$$

$$
Y_{T}^{\varepsilon}=\xi
$$

and $\left(\bar{Y}_{s}, \bar{Z}_{s}\right)$ is the mild solution of

$$
\begin{aligned}
d \bar{Y}_{t}= & -A \bar{Y}_{t} d t \\
& -\mathbb{E}^{\prime}\left[f\left(t,\left(\bar{Y}_{t}\right)^{\prime},\left(\bar{Z}_{t}\right)^{\prime}, \bar{Y}_{t}, \bar{Z}_{t}, \bar{u}_{t}\right)\right] d t \\
& +\bar{Z}_{t} d W(t), \quad t \in[0, T], \\
\bar{Y}_{T}=\xi . &
\end{aligned}
$$

By the definition of $u_{t}^{\varepsilon}$, according to (L2), we have

$$
\begin{aligned}
\mathbb{E}^{\prime}\left[f\left(s, Y^{\prime}(s), Z^{\prime}(s), Y(s), Z(s), u_{t}^{\varepsilon}\right)\right] \\
\quad \longrightarrow \mathbb{E}^{\prime}\left[f\left(s, Y^{\prime}(s), Z^{\prime}(s), Y(s), Z(s), \bar{u}_{t}\right)\right]
\end{aligned}
$$

in $L^{2}([0, T] ; H)$ as $\varepsilon \rightarrow 0$. Using the continuous dependence theorem Corollary 5 , we obtain

$$
\left(Y_{s}^{\varepsilon}, Z_{s}^{\varepsilon}\right) \longrightarrow\left(\bar{Y}_{s}, \bar{Z}_{s}\right) \quad \text { as } \varepsilon \longrightarrow 0 .
$$

Then,

$$
\begin{aligned}
\mathbb{E} \int_{t}^{T} \mathbb{E}^{\prime}\left[\left|K_{s}^{\varepsilon}\right|^{2} \int_{0}^{1}\left|f_{y}(s, \lambda)-f_{y}\left(u_{t}^{\varepsilon}\right)\right|^{2} d \lambda\right] d s \\
\leq C^{2} \lambda^{2} \mathbb{E} \int_{t}^{T}\left|K_{s}^{\varepsilon}\right|^{2} \\
\times \int_{0}^{1} \mathbb{E}^{\prime}\left[\left|\left(Y_{s}^{\varepsilon}-\bar{Y}_{s}\right)^{\prime}\right|^{2}+\left|\left(Z_{s}^{\varepsilon}-\bar{Z}_{s}\right)^{\prime}\right|^{2}\right. \\
\left.\times\left|Y_{s}^{\varepsilon}-\bar{Y}_{s}\right|^{2}+\left|Z_{s}^{\varepsilon}-\bar{Z}_{s}\right|^{2}\right] d \lambda d s
\end{aligned}
$$

$\longrightarrow 0, \quad$ as $\varepsilon \longrightarrow 0$. 
Combining (98) with (104), we finally have $\mathbb{E} \int_{t}^{T} \mathbb{E}^{\prime}\left[\left|I_{s}^{2}\right|^{2}\right] d s \rightarrow 0$, as $\varepsilon \rightarrow 0$.

The required result (93) follows by using the similar estimations for $I_{s}^{1}, I_{s}^{3}$, and $I_{s}^{4}$.

Note that $1-192 M_{A}^{2} C^{2} / \beta>0$ if $\beta$ is sufficiently large. Now to prove the desired result (84) it suffices to apply Gronwall's lemma and estimate (93) to inequality (92).

To deal with the state constraint (75), we need to recall the Ekeland variational principle.

Lemma 16 (Ekeland's variational principle, see [16, Lemma 4.1]). Let $(S, d)$ be a complete metric space and let $F(\cdot): S \rightarrow$ $\mathbb{R}$ be lower semicontinuous and bounded from below. If, for $\rho>$ 0 , there exists $u \in S$, such that

$$
F(u) \leq \inf _{v \in S} F(v)+\rho,
$$

then there exists $u^{\rho} \in S$, satisfying

(i) $F\left(u^{\rho}\right) \leq F(u)$,

(ii) $d\left(u^{\rho}, u\right) \leq \rho$,

(iii) $F\left(u^{\rho}\right) \leq F(v)+\rho \cdot d\left(u^{\rho}, u\right), \quad \forall v \neq u^{\rho}$.

Now fix $v \in \mathscr{U}_{\mathrm{ad}}$, and set

$$
\begin{array}{r}
S=\left\{\left.v(\cdot) \in \mathcal{U}_{\mathrm{ad}}\left|\sup _{0 \leq t \leq T} \mathbb{E}\right| v_{t}\right|^{2} \leq \mathbb{E}\left|\bar{u}_{t}\right|^{2}+|v|^{2}\right\}, \\
d(\bar{v}(\cdot), v(\cdot))=m\left\{t \in[0, T]|\mathbb{E}| \bar{v}_{t}-\left.v_{t}\right|^{2}>0\right\}, \\
\forall \bar{v}(\cdot), v(\cdot) \in S,
\end{array}
$$

where $m$ denotes the Lebesgue measure on $\mathbb{R}$.

The following result is proved as Proposition 4.1 in [16].

Lemma 17. $(S, d(\cdot, \cdot))$ is a complete metric space and $J^{\rho}$ is continuous and bounded on $S$, where

$$
\begin{aligned}
& J^{\rho}(v(\cdot))=\left\{(J(v(\cdot))-J(\bar{u}(\cdot))+\rho)^{2}\right. \\
&\left.+\left|\overline{\mathbb{E}} \int_{\mathcal{O}} \Phi\left(x,\left(Y_{0}(x)\right)^{\prime}, Y_{0}(x)\right) d x\right|^{2}\right\}^{1 / 2}, \\
& \forall v(\cdot) \in S
\end{aligned}
$$

and $(Y, Z)$ is the mild solution of (72) corresponding to the control $v$.

Now we consider the following free initial state optimal control problem:

$$
\inf _{v(\cdot) \in S} J^{\rho}(v(\cdot)) .
$$

It is easy to check that

$$
0 \leq \inf _{v(\cdot) \in S} J^{\rho}(v(\cdot)) \leq J^{\rho}(\bar{u}(\cdot))=\rho .
$$

According to Ekeland's variational principle, there exists a $u^{\rho}(\cdot) \in V$ such that

$$
\begin{aligned}
& \text { (i) } J^{\rho}\left(u^{\rho}(\cdot)\right) \leq \rho, \\
& \text { (ii) } d\left(u^{\rho}(\cdot), \bar{u}(\cdot)\right) \leq \rho, \\
& \text { (iii) } J^{\rho}\left(u^{\rho}(\cdot)\right) \leq J^{\rho}(v(\cdot))+\rho d\left(u^{\rho}(\cdot), \bar{u}(\cdot)\right), \quad \forall v(\cdot) \in S .
\end{aligned}
$$

Using the spike variation method, we can $\operatorname{construct} u^{\varepsilon \rho}(\cdot) \in S$ as follows:

$$
u_{t}^{\varepsilon \rho}= \begin{cases}v_{t}, & t \in[s, s+\varepsilon], \\ u_{t}^{\rho}, & t \in[0, T] \backslash[s, s+\varepsilon] .\end{cases}
$$

It is clear that $d\left(u^{\varepsilon \rho}(\cdot), u^{\rho}(\cdot)\right) \leq \varepsilon$. Let $\left(Y^{\varepsilon \rho}(\cdot), Z^{\varepsilon \rho}(\cdot)\right)$ (resp., $\left.\left(Y^{\rho}(\cdot), Z^{\rho}(\cdot)\right)\right)$ be the solution of (72) with respect to the control $u^{\varepsilon \rho}(\cdot)$ (resp., $\left.u^{\rho}(\cdot)\right)$. Following (82), $\left(K_{t}^{\varepsilon \rho}, Q_{t}^{\varepsilon \rho}\right)$ is the mild solution of

$$
\begin{aligned}
K_{t}^{\varepsilon \rho}=\int_{t}^{T} e^{A(s-t)} \mathbb{E}^{\prime} & \\
\quad & {\left[f_{y^{\prime}}\left(s, \Lambda_{s}^{\rho}, u_{s}^{\rho}\right)\left(K_{s}^{\varepsilon \rho}\right)^{\prime}+f_{y}\left(s, \Lambda_{s}^{\rho}, u_{s}^{\rho}\right) K_{s}^{\varepsilon \rho}\right.} \\
& \quad+f_{z^{\prime}}\left(s, \Lambda_{s}^{\rho}, u_{s}^{\rho}\right)\left(Q_{s}^{\varepsilon \rho}\right)^{\prime}+f_{z}\left(s, \Lambda_{s}^{\rho}, u_{s}^{\rho}\right) Q_{s}^{\varepsilon \rho} \\
& \left.\quad+\frac{1}{\varepsilon}\left(f\left(s, \Lambda_{s}^{\rho}, u_{s}^{\varepsilon \rho}\right)-f\left(s, \Lambda_{s}^{\rho}, u_{s}^{\rho}\right)\right)\right] d s \\
- & \int_{t}^{T} e^{A(s-t)} Q_{s}^{\varepsilon \rho} d W(s) .
\end{aligned}
$$

By Theorem 15, we know that

$$
\begin{gathered}
\lim _{\varepsilon \rightarrow 0} \mathbb{E}\left[\sup _{s \in[t, T]}\left|\frac{Y_{s}^{\varepsilon \rho}-Y_{s}^{\rho}}{\varepsilon}-K_{s}^{\varepsilon \rho}\right|^{2}\right]=0, \quad \forall t \in[0, T], \\
\lim _{\varepsilon \rightarrow 0} \mathbb{E} \int_{0}^{T}\left|\frac{Z_{s}^{\varepsilon \rho}-Z_{s}^{\rho}}{\varepsilon}-Q_{s}^{\varepsilon \rho}\right|^{2} d s=0 .
\end{gathered}
$$

The proof of the following proposition is technical but based on the arguments above and we omit it.

Proposition 18. One has

$$
\begin{aligned}
\frac{1}{\varepsilon} \overline{\mathbb{E}}\left[\Phi\left(\left(Y_{0}^{\varepsilon \rho}\right)^{\prime}, Y_{0}^{\varepsilon \rho}\right)-\Phi\left(\left(Y_{0}^{\rho}\right)^{\prime}, Y_{0}^{\rho}\right)\right] & \\
= & \overline{\mathbb{E}}\left[\Phi_{y^{\prime}}\left(\left(Y_{0}^{\rho}\right)^{\prime}, Y_{0}^{\rho}\right)\left(K_{0}^{\varepsilon \rho}\right)^{\prime}+\Phi_{y}\left(\left(Y_{0}^{\rho}\right)^{\prime}, Y_{0}^{\rho}\right) K_{0}^{\varepsilon \rho}\right] \\
& +o(\varepsilon),
\end{aligned}
$$




$$
\begin{aligned}
& \frac{1}{\varepsilon}\left(J\left(u^{\varepsilon \rho}\right)-J\left(u^{\rho}\right)\right) \\
& =\overline{\mathbb{E}}\left[g_{y^{\prime}}\left(\left(Y_{0}^{\rho}\right)^{\prime}, Y_{0}^{\rho}\right)\left(K_{0}^{\varepsilon \rho}\right)^{\prime}+g_{y}\left(\left(Y_{0}^{\rho}\right)^{\prime}, Y_{0}^{\rho}\right) K_{0}^{\varepsilon \rho}\right] \\
& +\Delta^{\varepsilon}+\frac{1}{\varepsilon} \mathbb{E} \int_{0}^{T} \mathbb{E}^{\prime}\left[h\left(s, \Lambda_{s}^{\rho}, u_{s}^{\varepsilon \rho}\right)\right. \\
& \left.\quad-h\left(s, \Lambda_{s}^{\rho}, u_{s}^{\rho}\right)\right] d s+o(\varepsilon),
\end{aligned}
$$

where

$$
\begin{aligned}
\Delta^{\varepsilon}=\mathbb{E} \int_{0}^{T} \mathbb{E}^{\prime} & h_{y^{\prime}}\left(s, \Lambda_{s}^{\rho}, u_{s}^{\varepsilon \rho}\right)\left(K_{s}^{\varepsilon \rho}\right)^{\prime} \\
& +h_{z^{\prime}}\left(s, \Lambda_{s}^{\rho}, u_{s}^{\varepsilon \rho}\right)\left(Q_{s}^{\varepsilon \rho}\right)^{\prime}+h_{y}\left(s, \Lambda_{s}^{\rho}, u_{s}^{\varepsilon \rho}\right) K_{s}^{\varepsilon \rho} \\
& \left.+h_{z}\left(s, \Lambda_{s}^{\rho}, u_{s}^{\varepsilon \rho}\right) Q_{s}^{\varepsilon \rho}\right] d s .
\end{aligned}
$$

5.3. Variational Inequality and Adjoint Equation. In this subsection, the adjoint process is introduced to deduce the variational inequality.

If we set $v(\cdot)=u^{\varepsilon \rho}(\cdot)$ in (111) and notice that $d\left(u^{\varepsilon \rho}(\cdot), u^{\rho}(\cdot)\right) \leq \varepsilon$, we get

$$
-\rho \leq \frac{1}{\varepsilon}\left(J^{\rho}\left(u^{\varepsilon \rho}(\cdot)\right)-J^{\rho}\left(u^{\rho}(\cdot)\right)\right) .
$$

By Lemma 17,

$$
\begin{aligned}
\frac{1}{\varepsilon}\left(J^{\rho}\left(u^{\varepsilon \rho}(\cdot)\right)-J^{\rho}\left(u^{\rho}(\cdot)\right)\right) & \\
= & \frac{\left(J^{\rho}\left(u^{\varepsilon \rho}(\cdot)\right)\right)^{2}-\left(J^{\rho}\left(u^{\rho}(\cdot)\right)\right)^{2}}{\varepsilon\left(J^{\rho}\left(u^{\varepsilon \rho}(\cdot)\right)+J^{\rho}\left(u^{\rho}(\cdot)\right)\right)} \\
= & \frac{J\left(u^{\varepsilon \rho}(\cdot)\right)+J\left(u^{\rho}(\cdot)\right)-2 J(\bar{u}(\cdot))+2 \rho}{J^{\rho}\left(u^{\varepsilon \rho}(\cdot)\right)+J^{\rho}\left(u^{\rho}(\cdot)\right)} \\
& \times \frac{J\left(u^{\varepsilon \rho}(\cdot)\right)-J\left(u^{\rho}(\cdot)\right)}{\varepsilon} \\
& +\frac{\overline{\mathbb{E}}\left[\Phi\left(\left(Y_{0}^{\varepsilon \rho}\right)^{\prime}, Y_{0}^{\varepsilon \rho}\right)\right]+\overline{\mathbb{E}}\left[\Phi\left(\left(Y_{0}^{\rho}\right)^{\prime}, Y_{0}^{\rho}\right)\right]}{J^{\rho}\left(u^{\varepsilon \rho}(\cdot)\right)+J^{\rho}\left(u^{\rho}(\cdot)\right)} \\
& \times \frac{\overline{\mathbb{E}}\left[\Phi\left(\left(Y_{0}^{\varepsilon \rho}\right)^{\prime}, Y_{0}^{\varepsilon \rho}\right)\right]-\overline{\mathbb{E}}\left[\Phi\left(\left(Y_{0}^{\rho}\right)^{\prime}, Y_{0}^{\rho}\right)\right]}{\varepsilon} \\
\longrightarrow & l_{1}^{\rho} \frac{J\left(u^{\varepsilon \rho}(\cdot)\right)-J\left(u^{\rho}(\cdot)\right)}{\varepsilon} \\
& +l_{2}^{\rho} \frac{\overline{\mathbb{E}}\left[\Phi\left(\left(Y_{0}^{\varepsilon \rho}\right)^{\prime}, Y_{0}^{\varepsilon \rho}\right)\right]-\overline{\mathbb{E}}\left[\Phi\left(\left(Y_{0}^{\rho}\right)^{\prime}, Y_{0}^{\rho}\right)\right]}{\varepsilon},
\end{aligned}
$$

where we set

$$
\begin{aligned}
& l_{1}^{\rho}=\frac{J\left(u^{\rho}(\cdot)\right)-J(\bar{u}(\cdot))+\rho}{J^{\rho}\left(u^{\rho}(\cdot)\right)}, \\
& l_{2}^{\rho}=\frac{\overline{\mathbb{E}}\left[\Phi\left(\left(Y_{0}^{\rho}\right)^{\prime}, Y_{0}^{\rho}\right)\right]}{J^{\rho}\left(u^{\rho}(\cdot)\right)}
\end{aligned}
$$

and use the limit

$$
\begin{aligned}
J\left(u^{\varepsilon \rho}(\cdot)\right) & \longrightarrow J\left(u^{\rho}(\cdot)\right), \\
\overline{\mathbb{E}}\left[\Phi\left(\left(Y_{0}^{\varepsilon \rho}\right)^{\prime}, Y_{0}^{\varepsilon \rho}\right)\right] & \longrightarrow \overline{\mathbb{E}}\left[\Phi\left(\left(Y_{0}^{\rho}\right)^{\prime}, Y_{0}^{\rho}\right)\right],
\end{aligned}
$$

as $\varepsilon \rightarrow 0$ according to (115).

As $\left|l_{1}^{\rho}\right|^{2}+\left|l_{2}^{\rho}\right|^{2}=1$ for all $\rho>0$, we know that there exists a subsequence of $\left\{l_{1}^{\rho}, l_{2}^{\rho}\right\}$ (still denoted by $\left\{l_{1}^{\rho}, l_{2}^{\rho}\right\}$ ) such that

$$
\begin{gathered}
\lim _{\rho \rightarrow 0}\left\{l_{1}^{\rho}, l_{2}^{\rho}\right\}=\left\{l_{1}, l_{2}\right\}, \\
\left|l_{1}\right|^{2}+\left|l_{2}\right|^{2}=1 .
\end{gathered}
$$

Combining (115), (117) with (118), we get

$$
\begin{aligned}
& -\rho \leq l_{1}^{\rho} \frac{J\left(u^{\varepsilon \rho}(\cdot)\right)-J\left(u^{\rho}(\cdot)\right)}{\varepsilon} \\
& +l_{2}^{\rho} \frac{\overline{\mathbb{E}}\left[\Phi\left(\left(Y_{0}^{\varepsilon \rho}\right)^{\prime}, Y_{0}^{\varepsilon \rho}\right)\right]-\overline{\mathbb{E}}\left[\Phi\left(\left(Y_{0}^{\rho}\right)^{\prime}, Y_{0}^{\rho}\right)\right]}{\varepsilon} \\
& =l_{1}^{\rho} \Delta^{\varepsilon}+l_{1}^{\rho} \overline{\mathbb{E}}\left[g_{y^{\prime}}\left(\left(Y_{0}^{\rho}\right)^{\prime}, Y_{0}^{\rho}\right)\left(K_{0}^{\varepsilon \rho}\right)^{\prime}\right. \\
& \left.+g_{y}\left(\left(Y_{0}^{\rho}\right)^{\prime}, Y_{0}^{\rho}\right) K_{0}^{\varepsilon \rho}\right] \\
& +\frac{l_{1}^{\rho}}{\varepsilon} \mathbb{E} \int_{0}^{T} \mathbb{E}^{\prime}\left[h\left(s, \Lambda_{s}^{\rho}, u_{s}^{\varepsilon \rho}\right)-h\left(s, \Lambda_{s}^{\rho}, u_{s}^{\rho}\right)\right] d s \\
& +l_{2}^{\rho} \overline{\mathbb{E}}\left[\Phi_{y^{\prime}}\left(\left(Y_{0}^{\rho}\right)^{\prime}, Y_{0}^{\rho}\right)\left(K_{0}^{\varepsilon \rho}\right)^{\prime}\right. \\
& \left.+\Phi_{y}\left(\left(Y_{0}^{\rho}\right)^{\prime}, Y_{0}^{\rho}\right) K_{0}^{\varepsilon \rho}\right]+\left(l_{1}^{\rho}+l_{2}^{\rho}\right) o(\varepsilon) .
\end{aligned}
$$

Next, we introduce the adjoint equation corresponding to variational equation (113), whose solution is denoted by $P^{\rho}(t)$ :

$$
\begin{aligned}
& d P^{\rho}(t) \\
& =A^{*} P^{\rho}(t) d t \\
& +\mathbb{E}^{\prime}\left[f_{y^{\prime}}\left(t, \Lambda_{t}^{\rho}, u_{t}^{\rho}\right)\left(P^{\rho}(t)\right)^{\prime}\right. \\
& +f_{y}\left(t, \Lambda_{t}^{\rho}, u_{t}^{\rho}\right) P^{\rho}(t)+l_{1}^{\rho} h_{y^{\prime}}\left(t, \Lambda_{t}^{\rho}, u_{t}^{\rho}\right) \\
& \left.+l_{1}^{\rho} h_{y}\left(t, \Lambda_{t}^{\rho}, u_{t}^{\rho}\right)\right] d t
\end{aligned}
$$




$$
\begin{aligned}
+\mathbb{E}^{\prime}\left[f_{z^{\prime}}\left(t, \Lambda_{t}^{\rho}, u_{t}^{\rho}\right)\left(P^{\rho}(t)\right)^{\prime}+f_{z}\left(t, \Lambda_{t}^{\rho}, u_{t}^{\rho}\right) P^{\rho}(t)\right. \\
\left.+l_{1}^{\rho} h_{z^{\prime}}\left(t, \Lambda_{t}^{\rho}, u_{t}^{\rho}\right)+l_{1}^{\rho} h_{z}\left(t, \Lambda_{t}^{\rho}, u_{t}^{\rho}\right)\right] d W(t), \\
P^{\rho}(0)=l_{1}^{\rho} \overline{\mathbb{E}}\left[g_{y^{\prime}}\left(\left(Y_{0}^{\rho}(x)\right)^{\prime}, Y_{0}^{\rho}(x)\right)\right. \\
\left.+g_{y}\left(\left(Y_{0}^{\rho}(x)\right)^{\prime}, Y_{0}^{\rho}(x)\right)\right] \\
+l_{2}^{\rho} \overline{\mathbb{E}}\left[\Phi_{y^{\prime}}\left(\left(Y_{0}^{\rho}(x)\right)^{\prime}, Y_{0}^{\rho}(x)\right)\right. \\
\left.+\Phi_{y}\left(\left(Y_{0}^{\rho}(x)\right)^{\prime}, Y_{0}^{\rho}(x)\right)\right],
\end{aligned}
$$

where $A^{*}$ is the $L^{2}(\mathcal{O})$-adjoint operator of $A$. Under assumptions (L1)-(L3), this is a linear mean-field SEE with bounded coefficients. An application of Theorem 11 implies that it has a unique adapted mild solution such that $P^{\rho}(t) \in \delta_{\mathbb{F}}^{2}([0, T] ; K)$.

When $\rho \rightarrow 0$, according to Corollaries 5 and 13, $P^{\rho}(t)$ converges to $\bar{P}(t)$, where

$$
\bar{P}(t) \in \mathcal{S}_{\mathbb{F}}^{2}([0, T] ; K)
$$

is the solution of the following equation:

$$
\begin{array}{r}
\bar{P}(t)=l_{1} \overline{\mathbb{E}}\left[g_{y^{\prime}}\left(\bar{Y}_{0}^{\prime}, \bar{Y}_{0}\right)+g_{y}\left(\bar{Y}_{0}^{\prime}, \bar{Y}_{0}\right)\right] \\
+l_{2} \overline{\mathbb{E}}\left[\Phi_{y^{\prime}}\left(\bar{Y}_{0}^{\prime}, \bar{Y}_{0}\right)+\Phi_{y}\left(\bar{Y}_{0}^{\prime}, \bar{Y}_{0}\right)\right] \\
+\int_{0}^{t} e^{A^{*}(t-s)} \mathbb{E}^{\prime}\left[f_{y^{\prime}}(s) \bar{P}^{\prime}(s)+f_{y}(s) \bar{P}(s)\right. \\
\left.+l_{1} h_{y^{\prime}}(s)+l_{1} h_{y}(s)\right] d s \\
+\int_{0}^{t} e^{A^{*}(t-s)} \mathbb{E}^{\prime}\left[f_{z^{\prime}}(s) \bar{P}^{\prime}(s)+f_{z}(s) \bar{P}(s)\right. \\
\left.+l_{1} h_{z^{\prime}}(s)+l_{1} h_{z}(s)\right] d W(s) .
\end{array}
$$

The following proposition, which formally follows from Proposition 18, gives the relation between $P^{\rho}(t)$ and $K_{t}^{\varepsilon \rho}$.

Proposition 19. Consider the following:

$$
\begin{aligned}
\mathbb{E}[ & \left.P^{\rho}(0) K_{0}^{\varepsilon \rho}\right] \\
=\mathbb{E} \int_{0}^{T} & \left\{\frac{1}{\varepsilon} P^{\rho}(s) \mathbb{E}^{\prime}\left[f\left(s, \Lambda_{s}^{\rho}, u_{s}^{\varepsilon \rho}\right)-f\left(s, \Lambda_{s}^{\rho}, u_{s}^{\rho}\right)\right]\right. \\
& -l_{1}^{\rho} K_{s}^{\varepsilon \rho} \mathbb{E}^{\prime}\left[h_{y^{\prime}}\left(s, \Lambda_{s}^{\rho}, u_{s}^{\rho}\right)+h_{y}\left(s, \Lambda_{s}^{\rho}, u_{s}^{\rho}\right)\right] \\
& \left.-l_{1}^{\rho} Q_{s}^{\varepsilon \rho} \mathbb{E}^{\prime}\left[h_{z^{\prime}}\left(s, \Lambda_{s}^{\rho}, u_{s}^{\rho}\right)+h_{z}\left(s, \Lambda_{s}^{\rho}, u_{s}^{\rho}\right)\right]\right\} d s .
\end{aligned}
$$

The following theorem constitutes the main contribution of this section, the maximum principle for the BSPDE control system.

Theorem 20. Let assumptions (L1)-(L3) hold. Suppose $\bar{u}(\cdot)$ is an optimal control and $(\bar{Y}(\cdot), \bar{Z}(\cdot))$ is the corresponding optimal state trajectory for the BSPDE control systems (72) and (73) with the initial state constraint (75). Then there exists $\bar{P}(t) \in$ $\mathcal{S}_{\mathbb{F}}^{2}([0, T] ; K)$ which satisfies (125), such that

$$
\begin{array}{r}
\mathscr{H}\left(t, \bar{Y}_{t}^{\prime}, \bar{Z}_{t}^{\prime}, \bar{Y}_{t}, \bar{Z}_{t}, v_{t}, \bar{P}(t)\right) \\
\geq \mathscr{H}\left(t, \bar{Y}_{t}^{\prime}, \bar{Z}_{t}^{\prime}, \bar{Y}_{t}, \bar{Z}_{t}, \bar{u}_{t}, \bar{P}(t)\right), \\
\text { a.e., a.s. } \forall v \in \mathscr{U}_{a d},
\end{array}
$$

where $\mathscr{H}:[0, T] \times H \times \mathscr{L}(\Gamma, H) \times H \times \mathscr{L}(\Gamma, H) \times U \times K \rightarrow \mathbb{R}$ is the Hamiltonian function defined by

$$
\begin{aligned}
\mathscr{H}(t, \tilde{y}, \widetilde{z}, y, z, v, p)= & l_{1} h(t, \tilde{y}, \widetilde{z}, y, z, v) \\
& +p f(t, \tilde{y}, \widetilde{z}, y, z, v) .
\end{aligned}
$$

Proof. By (122) and Proposition 19, we obtain

$$
\begin{aligned}
&-\rho \leq \frac{1}{\varepsilon} \mathbb{E} \int_{0}^{T} P^{\rho}(s) \mathbb{E}^{\prime} {\left[f\left(s, \Lambda_{s}^{\rho}, u_{s}^{\varepsilon \rho}\right)\right.} \\
&\left.-f\left(s, \Lambda_{s}^{\rho}, u_{s}^{\rho}\right)\right] d s \\
&+\frac{l_{1}^{\rho}}{\varepsilon} \mathbb{E} \int_{0}^{T} \mathbb{E}^{\prime}\left[h\left(s, \Lambda_{s}^{\rho}, u_{s}^{\varepsilon \rho}\right)\right. \\
&\left.-h\left(s, \Lambda_{s}^{\rho}, u_{s}^{\rho}\right)\right] d s+\left(l_{1}^{\rho}+l_{2}^{\rho}\right) o(\varepsilon) .
\end{aligned}
$$

Letting $\varepsilon \rightarrow 0^{+}$in (129), we derive, for a.e. $\tau \in[0, T]$,

$$
\begin{aligned}
-\rho \leq & l_{1}^{\rho} \mathbb{E}^{\prime}\left[h\left(\tau, \Lambda_{\tau}^{\rho}, v_{\tau}\right)-h\left(\tau, \Lambda_{\tau}^{\rho}, u_{\tau}^{\rho}\right)\right] \\
& +P^{\rho}(s) \mathbb{E}^{\prime}\left[f\left(\tau, \Lambda_{\tau}^{\rho}, v_{\tau}\right)-f\left(\tau, \Lambda_{\tau}^{\rho}, u_{\tau}^{\rho}\right)\right],
\end{aligned}
$$

for all $v \in \mathcal{U}_{\text {ad }}$. result.

Finally, taking $\rho \rightarrow 0$ in (130), we derive the desired

Remark 21. We note that if the coefficients do not depend explicitly on the marginal law of the underlying diffusion, the result reduces to the classical case, that is, the SMP for BSPDEs without mean-field term.

Remark 22. When we remove the initial state constraint (75), we obtain the general maximum principle for the mean-field BSPDEs system (i.e., without the constraint) with $l_{1}=1$.

5.4. Application: A Backward Linear Quadratic Control Problem. Now, we apply our maximum principle to solve an LQ problem. For notational simplicity, we restrict ourselves to the free case (i.e., without the initial state constraint (75)), the general case being handled in a similar way. 
Consider the following problem:

$$
J(u)=\frac{1}{2} \mathbb{E}\left[(Y(0))^{2}\right]+\frac{1}{2} \mathbb{E} \int_{0}^{T}\left\{N v^{2}(t)\right\} d t \longrightarrow \min
$$

subject to

$$
\begin{aligned}
& d Y(t)=- A Y(t) d t \\
&-\{B Y(t)+\widetilde{B} \mathbb{E}[Y(t)] \\
&+C v(t)+D Z(t)+\widetilde{D} \mathbb{E}[Z(t)]\} d t \\
&+ Z(t) d W(t), \\
& Y(T)=\xi, \quad t \in[0, T],
\end{aligned}
$$

where $A$ is a partial differential operator satisfying condition (L1) and $B, \widetilde{B}, C, D, \widetilde{D}$, and $N$ are bounded and deterministic constants. We also assume that $N>0$ and $v \in L_{\mathbb{F}}^{2}(0, T ; U)$. It is easy to verify that BSPDE (132) admits a unique mild solution $(Y(t), Z(t))$.

$P(t)$, the adjoint process of state equation (132), is the solution of

$$
\begin{gathered}
d P(t)=A^{*} P(t) d t+\{B P(t)+\widetilde{B} \mathbb{E}[P(t)]\} d t \\
+\{D P(t)+\widetilde{D} \mathbb{E}[P(t)]\} d W(t), \\
P(0)=Y(0) .
\end{gathered}
$$

Let $\bar{u}(\cdot)$ be an optimal control, and let $(\bar{Y}(\cdot), \bar{Z}(\cdot))$ be the corresponding state process. By maximum principle of Theorem 20 (note that $l_{1}=1$ in this problem),

$$
\frac{1}{2} N v^{2}(t)+P(t) C v(t) \geq \frac{1}{2} N \bar{u}^{2}(t)+P(t) C \bar{u}(t)
$$

for all $v \in U_{\text {ad }}$ since the state equation has the form (132). This in turn implies

$$
\bar{u}(t)=-\frac{C}{N} P(t) .
$$

It is clear that (131) is a positive quadratic functional of control because of $N>0$. Hence, an optimal control exists. The candidate optimal control (135) is indeed an optimal control of this LQ problem for it is the only control which satisfies the maximum principle.

Next, we want to obtain a more explicit representation of the optimal control (135) from the state equation (132). Substituting (135) into state equation (132) yields

$$
\begin{aligned}
& d Y(t)=-A Y(t) d t \\
& -\left\{B Y(t)+\widetilde{B} \mathbb{E}[Y(t)]-\frac{C^{2}}{N} P(t)\right. \\
& +D Z(t)+\widetilde{D} \mathbb{E}[Z(t)]\} d t+Z(t) d W(t), \\
& Y(T)=\xi, \quad t \in[0, T] .
\end{aligned}
$$

Combining the above equation with (133), we obtain the following related feedback control system:

$$
\begin{gathered}
d Y(t)=-A Y(t) d t \\
-\left\{B Y(t)+\widetilde{B} \mathbb{E}[Y(t)]-\frac{C^{2}}{N} P(t)\right. \\
+D Z(t)+\widetilde{D} \mathbb{E}[Z(t)]\} d t+Z(t) d W(t), \\
Y(T)=\xi \\
d P(t)=A^{*} P(t) d t+\{B P(t)+\widetilde{B} \mathbb{E}[P(t)]\} d t \\
+\{D P(t)+\widetilde{D} \mathbb{E}[P(t)]\} d W(t), \\
P(0)=Y(0) .
\end{gathered}
$$

Looking at the terminal condition of $P(t)$ in (133) and considering the mean-field type of (132), it is reasonable to conjecture that $P(t)$ has the following form:

$$
P(t)=\varphi(t) Y(t)+\phi(t) \mathbb{E}[Y(t)],
$$

where $\varphi(t), \phi(t)$ are deterministic differential functions which will be specified below. Moreover, $\varphi(0)=1, \phi(0)=0$.

Inserting this form into adjoint equation (133) and noticing that $Y(t)$ satisfies (136), we can compare the coefficients of $d t$ and $d W(t)$ to obtain the following equation:

$$
\begin{aligned}
2 \varphi(t) & A Y(t)+2 \phi(t) A \mathbb{E}[Y(t)]+B \varphi(t) Y(t) \\
+ & 2(B \phi(t)+\widetilde{B} \varphi(t)+\widetilde{B} \phi(t)) \mathbb{E}[Y(t)] \\
= & -\varphi(t)\left\{B Y(t)-\frac{C^{2}}{N} \varphi(t) Y(t)+D Z(t)\right\} \\
+ & Y(t) \frac{d \varphi(t)}{d t}+\mathbb{E}[Y(t)] \frac{d \phi(t)}{d t} \\
+ & \left(\phi^{2}(t) \frac{C^{2}}{N}+2 \frac{C^{2}}{N} \varphi(t) \phi(t)\right) \mathbb{E}[Y(t)] \\
- & (D \phi(t)+\widetilde{D} \phi(t)+\widetilde{D} \varphi(t)) \mathbb{E}[Z(t)], \\
\varphi(t) Z(t)=D \varphi(t) Y(t) & +(D \phi(t)+\widetilde{D} \varphi(t)+\widetilde{D} \phi(t)) \mathbb{E}[Y(t)] .
\end{aligned}
$$

Then, subtracting $Z(t)$ we have

$$
\begin{gathered}
2 \varphi(t) A Y(t)+2 \phi(t) A \mathbb{E}[Y(t)]+B \varphi(t) Y(t) \\
+2(B \phi(t)+\widetilde{B} \varphi(t)+\widetilde{B} \phi(t)) \mathbb{E}[Y(t)]
\end{gathered}
$$




$$
\begin{aligned}
= & \varphi(t)\left\{-B+\frac{C^{2}}{N} \varphi(t)-D^{2}\right\} Y(t) \\
& +Y(t) \frac{d \varphi(t)}{d t}+\mathbb{E}[Y(t)] \frac{d \phi(t)}{d t} \\
& +\left(\phi^{2}(t) \frac{C^{2}}{N}+2 \frac{C^{2}}{N} \varphi(t) \phi(t)\right) \mathbb{E}[Y(t)] \\
& -\frac{1}{\varphi(t)}(D \phi(t)+\widetilde{D} \varphi(t)+\widetilde{D} \phi(t))^{2} \mathbb{E}[Y(t)] \\
& -2 D(D \phi(t)+\widetilde{D} \varphi(t)+\widetilde{D} \phi(t)) \mathbb{E}[Y(t)] .
\end{aligned}
$$

Comparing the coefficients of $Y(t)$ and $\mathbb{E}[Y(t)]$, respectively, we get

$$
\begin{gathered}
\frac{d}{d t} \varphi(t)=2 A^{*} \varphi(t)+2 B \varphi(t)+D^{2} \varphi(t)-\frac{C^{2}}{N} \varphi^{2}(t), \\
\varphi(0)=1, \\
\frac{d}{d t} \phi(t)=2 A^{*} \phi(t)+\left(\frac{(D+\widetilde{D})^{2}}{\varphi(t)}-\frac{C^{2}}{N}\right) \phi^{2}(t) \\
+2 R(t) \phi(t)+\left(\widetilde{D}^{2}+2 \widetilde{B}+2 D \widetilde{D}\right) \varphi(t), \\
\phi(0)=0,
\end{gathered}
$$

where $R(t)=B+\widetilde{B}+(D+\widetilde{D})^{2}-\left(C^{2} / N\right) \varphi(t)$.

We solve (141) to get

$$
\begin{gathered}
\varphi(t)=\left(e^{-\left(2 A^{*}+2 B+D^{2}\right) t}\left(1-\frac{C^{2}}{N\left(2 A^{*}+2 B+D^{2}\right)}\right)\right. \\
\left.+\frac{C^{2}}{N\left(2 A^{*}+2 B+D^{2}\right)}\right)^{-1} .
\end{gathered}
$$

Then (142) exists, a unique solution from the classical Riccati equation theory.

We now conclude the above discussions in the following result.

Theorem 23. For one's linear quadratic stochastic partial differential control problem (131)-(132), the unique optimal control $\bar{u}(\cdot) \in U_{a d}$ is given by

$$
\bar{u}(t)=-\frac{C}{N}(\varphi(t) Y(t)+\phi(t) \mathbb{E}[Y(t)]),
$$

with $\varphi(t)$ satisfying (143) and $\phi(t)$ solving (142).

\section{Conflict of Interests}

The authors declare that they have no conflict of interests regarding the publication of this paper.

\section{Acknowledgment}

This research is partially supported by the Natural Science Foundation of China under Grant no. 11301310.

\section{References}

[1] Y. Hu and S. Peng, "Adapted solution of a backward semilinear stochastic evolution equation," Stochastic Analysis and Applications, vol. 9, no. 4, pp. 445-459, 1991.

[2] N. I. Mahmudov and M. A. McKibben, "On backward stochastic evolution equations in Hilbert spaces and optimal control," Nonlinear Analysis, vol. 67, no. 4, pp. 1260-1274, 2007.

[3] M. Fuhrman and G. Tessitore, "Nonlinear Kolmogorov equations in infinite dimensional spaces: the backward stochastic differential equations approach and applications to optimal control," The Annals of Probability, vol. 30, no. 3, pp. 1397-1465, 2002.

[4] M. Fuhrman and G. Tessitore, "Infinite horizon backward stochastic differential equations and elliptic equations in Hilbert spaces," Annals of Probability, vol. 32, no. 1B, pp. 607660, 2004.

[5] X. Mao, "Adapted solutions of backward stochastic differential equations with non-Lipschitz coefficients," Stochastic Processes and their Applications, vol. 58, no. 2, pp. 281-292, 1995.

[6] J. Lasry and P. Lions, "Mean field games," Japanese Journal of Mathematics, vol. 2, no. 1, pp. 229-260, 2007.

[7] R. Buckdahn, J. Li, and S. Peng, "Mean-field backward stochastic differential equations and related partial differential equations," Stochastic Processes and their Applications, vol. 119, no. 10, pp. 3133-3154, 2009.

[8] D. Andersson and B. Djehiche, "A maximum principle for SDEs of mean-field type," Applied Mathematics and Optimization, vol. 63, no. 3, pp. 341-356, 2011.

[9] R. Buckdahn, B. Djehiche, J. Li, and S. Peng, "Mean-field backward stochastic differential equations: a limit approach," The Annals of Probability, vol. 37, no. 4, pp. 1524-1565, 2009.

[10] R. Xu, "Mean-field backward doubly stochastic differential equations and related SPDEs," Boundary Value Problems, vol. 2012, article 114, 2012.

[11] R. Buckdahn, B. Djehiche, and J. Li, "A general stochastic maximum principle for SDEs of mean-field type," Applied Mathematics and Optimization, vol. 64, no. 2, pp. 197-216, 2011.

[12] J. Li, "Stochastic maximum principle in the mean-field controls," Automatica, vol. 48, no. 2, pp. 366-373, 2012.

[13] G. Wang, C. Zhang, and W. Zhang, "Stochastic maximum principle for mean-field type optimal control under partial information," IEEE Transactions on Automatic Control, vol. 59, no. 2, pp. 522-528, 2014.

[14] M. Hafayed, "A mean-field necessary and sufficient conditions for optimal singular stochastic control," Communications in Mathematics and Statistics, vol. 1, no. 4, pp. 417-435, 2013.

[15] M. Hafayed, "A mean-field maximum principle for optimal control of forward-backward stochastic differential equations with Poisson jump processes," International Journal of Dynamics and Control, vol. 1, no. 4, pp. 300-315, 2013.

[16] Y. Hu and S. Peng, "Maximum principle for semilinear stochastic evolution control systems," Stochastics and Stochastics Reports, vol. 33, no. 3-4, pp. 159-180, 1990.

[17] M. Fuhrman, Y. Hu, and G. Tessitore, "Stochastic maximum principle for optimal control of SPDEs," Comptes Rendus Mathematique, vol. 350, no. 13-14, pp. 683-688, 2012. 


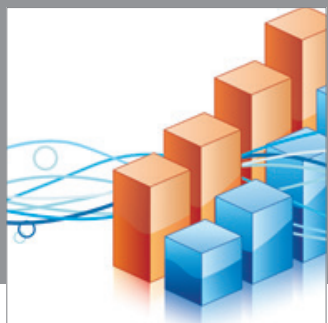

Advances in

Operations Research

mansans

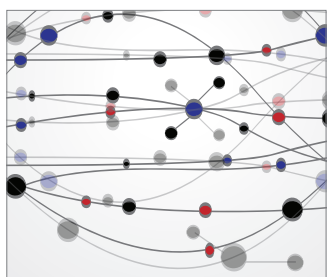

The Scientific World Journal
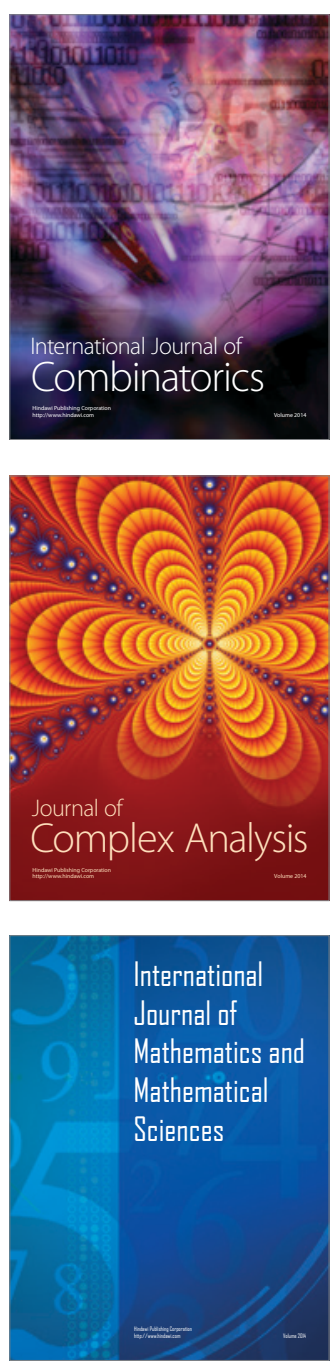
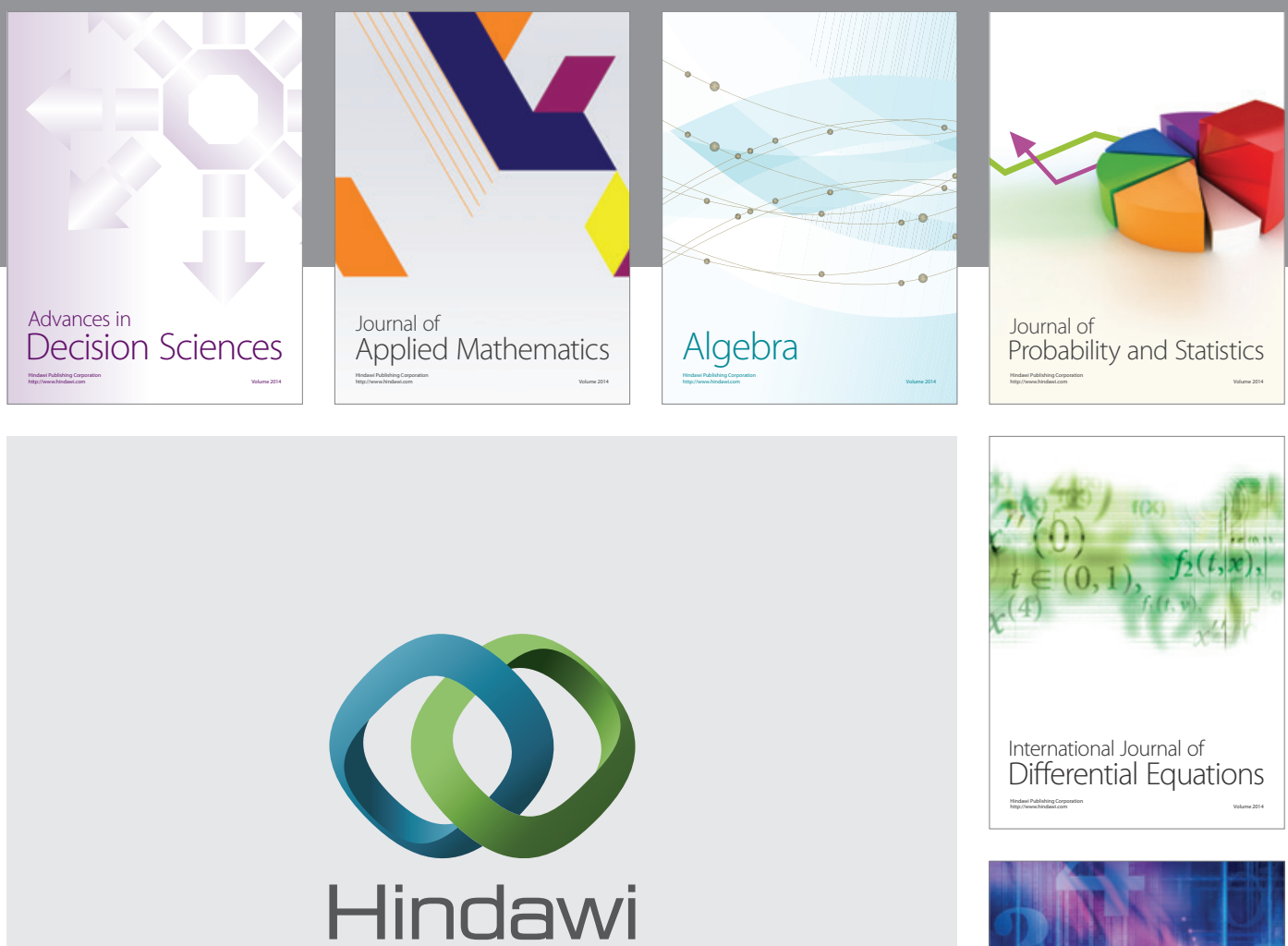

Submit your manuscripts at http://www.hindawi.com
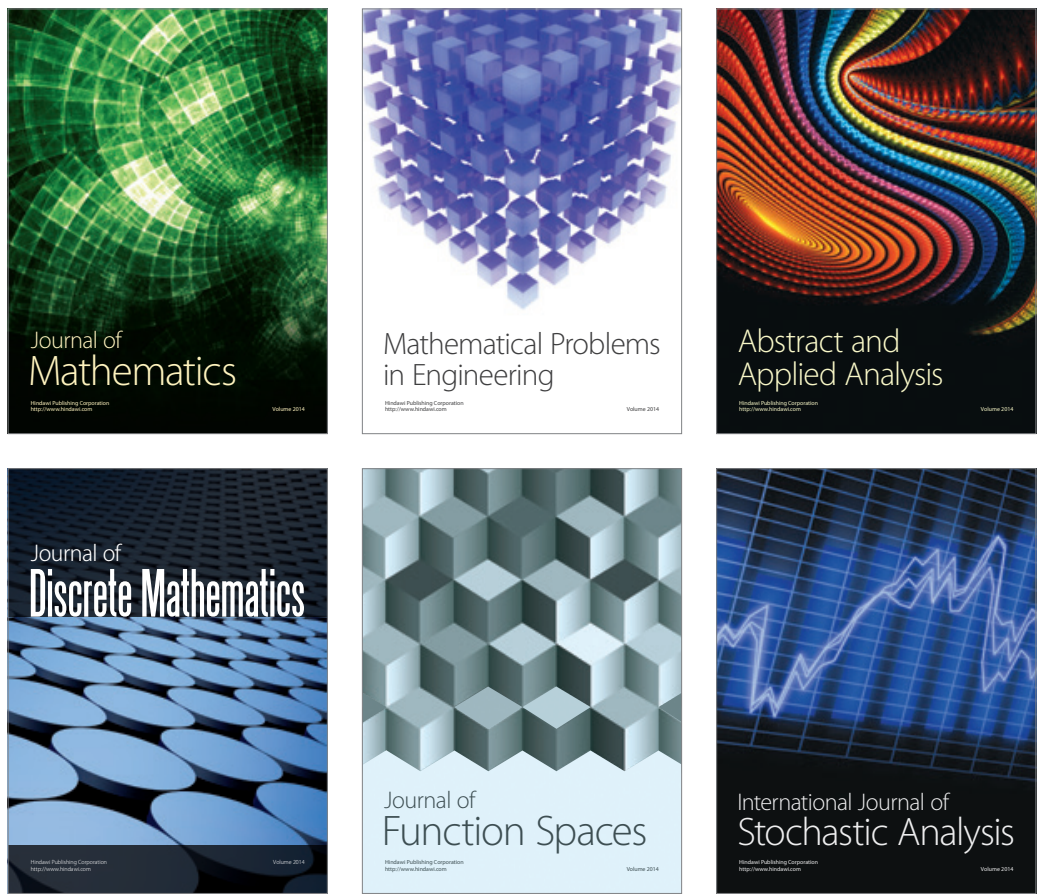

Journal of

Function Spaces

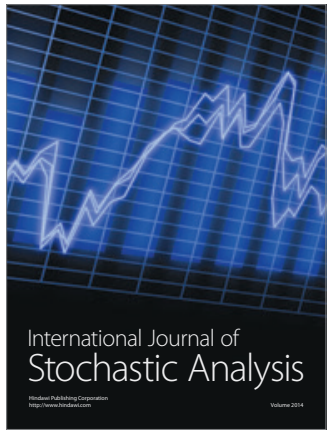

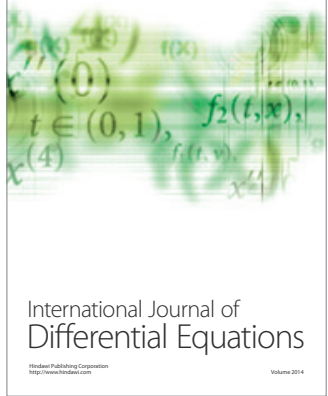
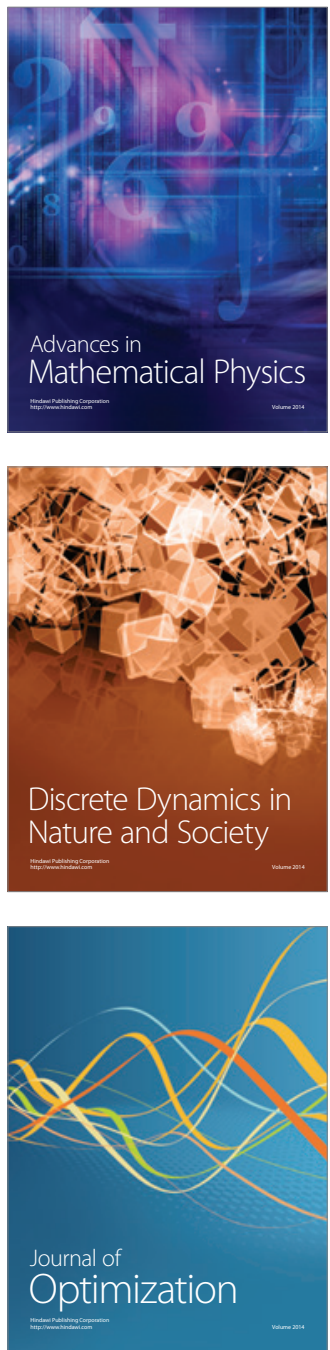